Homology, Homotopy and Applications, vol.9(1), 2007, pp.75-106

\title{
NOTES ON 2-GROUPOIDS, 2-GROUPS AND CROSSED MODULES
}

\author{
BEHRANG NOOHI
}

\author{
(communicated by J.F. Jardine)
}

\begin{abstract}
This paper contains some basic results on 2-groupoids, with special emphasis on computing derived mapping 2-groupoids between 2-groupoids and proving their invariance under strictification. Some of the results proven here are presumably folklore (but do not appear in the literature to the author's knowledge) and some of the results seem to be new. The main technical tool used throughout the paper is the Quillen model structure on the category of 2-groupoids introduced by Moerdijk and Svensson.
\end{abstract}

\section{Introduction}

Crossed modules, invented by J.H.C. Whitehead in the early twentieth century, are algebraic models for (connected) homotopy 2-types (i.e. connected spaces with no homotopy group in degrees above 2), in much the same way that groups are algebraic models for homotopy 1-types. It is a standard fact that a crossed module is essentially the same thing as a 2-group (that is, a 2-category with one object in which all 1-morphisms and all 2-morphisms are strictly invertible). This is made precise by establishing an equivalence between the category of crossed modules and the category of 2-groups (Section 3). In particular, 2-groups can also be used to model connected 2-types. More generally, 2-groupoids can be used to model arbitrary (i.e. not necessarily connected) 2-types.

But what do we really mean when we say 2 -groupoids model homotopy 2 -types?

According to Whitehead, to a connected homotopy 2-type one can associate a crossed module and from this crossed module one can recover the original 2-type. More generally, to any 2-type one can associate a 2-groupoid (the Whitehead 2groupoid) and from this 2-groupoid one can recover the original 2-type. One should keep in mind, however, that understanding 2-types is not only about understanding a single space with vanishing $\pi_{i}, i \geqslant 3$, but also about understanding mapping spaces between such spaces. So one may ask: is the category of 2-groupoids rich enough to provide us with such information? For instance, given 2-groupoids $\mathfrak{G}$ and $\mathfrak{H}$, can we capture the set of homotopy classes of maps between the corresponding 2-types from the 2-groupoids of morphisms between $\mathfrak{G}$ and $\mathfrak{H}$ ?

Received December 22, 2005, revised September 23, 2006; published on November 23, 2006; minor corrections January 5, 2007.

2000 Mathematics Subject Classification: 18D05, 18G50, 55U40.

Key words and phrases: homology, homotopy, 2-group, 2-groupoid, crossed module, 2-type, lax 2 -functors, weak 2-functors.

Copyright (c) 2006, International Press. Permission to copy for private use granted. 
The answer to the latter question is indeed no. There are usually far too few 2-groupoid morphisms to produce all homotopy classes. As a consequence, to have correct algebraic models for mapping spaces between 2-types, one needs to resort to more sophisticated tools.

There are two standard ways to go about this problem. One is to enlarge the set of morphisms by taking into account weak morphisms between 2-groupoids. The other is to endow the category of 2-groupoids with a model structure and use a cofibrant replacement procedure to define derived mapping spaces. There seems to be a common understanding among experts how these things should be done and agreement that both approaches yield essentially the same results. But to my knowledge, there are not many, if any, places in the literature where one can look for precise statements, let alone proofs.

This article is an attempt to fill this gap. The goal is to present various models for the mapping space between two given 2-groupoids (or weak 2-groupoids) and to verify that such models are naturally homotopy equivalent and that they provide algebraic models for the mapping space between the corresponding 2-types. We will also do the pointed version of these results. The results of this paper are used in [No] to provide yet another model for the space of weak morphisms between two crossed modules, which is very simple and handy and has interesting applications to the study of group actions on stacks.

This paper is organized as follows. There are essentially three parts, each one introducing a certain degree of "weakness" to the previous one!

In the first part, which includes up to Section 7 , we are concerned with strict 2-groupoids and strict functors between them, and we try to understand the derived mapping spaces (read, 2-groupoids) between 2-groupoids. (We will, however, already need to use weak transformations in this section, as strict transformation are not homotopically correct in this context.) Our main technical tool is the MoerdijkSvensson model structure, and its monoidal strengthening developed in [La04].

In the second part of the paper, Section 7 and Section 8, we allow weak 2functors into the picture, and study the corresponding simplicial mapping spaces. The point is that allowing this extra flexibility lets us get away with not having to make cofibrant replacements when computing derived mapping spaces. That is, the 2 -groupoid of weak 2-functors, weak 2-transformations and modification between arbitrary 2-groupoids $\mathfrak{H}$ and $\mathfrak{G}$ has the correct homotopy type and is homotopy equivalent to the derived mapping space.

In the third part of the paper, Section 9, we bring in weak 2-groupoids. We see that introducing weak 2-groupoids essentially does not add anything to the homotopy theory. Put differently, we can always replace weak 2-groupoids with strict ones without losing any homotopy theoretic information, and this strictification does not alter derived mapping spaces.

Since the author's ultimate goal is to apply these results to 2-groups and crossed modules, we also develop a pointed version of the theory, parallel to the unpointed one, and explain briefly how the pointed theory can be translated to the language of crossed modules. 


\section{Acknowledgements}

I would like to thank the Max-Planck-Institut für Mathematik, Bonn, where the research for this work was done, for providing pleasant working conditions. I thank Joachim Kock for reading the manuscript and making many useful remarks, Fernando Muro for many useful discussions and for explaining Andy Tonks' results to me, and Bertrand Toën for pointing out the work of Steve Lack [La04]. I would also like to thank Tim Porter for useful comments on an earlier version of this paper. Thanks are due to an anonymous referee for suggesting inclusion of the discussion of monoidal properties of the functors $N$ and $W$ in $\S 7.3$.

\section{Contents}

$\begin{array}{lll} & \text { Introduction } & 75\end{array}$

$\begin{array}{lll}2 & \text { Notations and terminology } & 78\end{array}$

3 Quick review of 2-groups and crossed modules $\quad \mathbf{7 9}$

3.1 Quick review of 2-groups . . . . . . . . . . . . . . . . 79

3.2 Quick review of crossed modules . . . . . . . . . . . . . 79

3.3 Equivalence of 2-groups and crossed modules . . . . . . . . . . . 80

4 2-categories and 2-groupoids $\quad \mathbf{8 0}$

4.1 Strict transformations between 2 -functors . . . . . . . . . . . . . . . 81

4.2 Weak transformations between 2 -functors . . . . . . . . . . . . 82

4.3 Nerve of a 2-category . . . . . . . . . . . . . . . . 83

5 Closed model structure on 2Gpd $\quad \mathbf{8 4}$

5.1 Monoidal closed model structure on 2Gpd . . . . . . . . . . . 86

6 The pointed category $2 \mathrm{Gpd}_{*}$ and application to crossed modules 87 6.1 Translation to the language of crossed modules . . . . . . . . . 88

7 Weak 2-functors between 2-categories $\quad 89$

7.1 Transformations between weak functors . . . . . . . . . . . . . 90

7.2 Nerves of weak functors . . . . . . . . . . . . . . . . . . 90

7.3 Monoidal properties of $N$ and $W \ldots \ldots$. . . . . . . . . . . 94

8 Pointed weak functors and application to crossed modules $\quad 95$

9 Weak 2-groupoids $\quad 97$

9.1 Weak functors between weak 2-categories, and transformations . . . 99

9.2 Nerve of a weak 2-groupoid . . . . . . . . . . . . . . . . . . . . . 99

9.3 Strictifying weak 2 -groupoids . . . . . . . . . . . . . . . 102 
Homology, Homotopy and Applications, vol. 9(1), 2007

\section{Notations and terminology}

A 2-functor between 2-categories means a strict 2-functor. We sometimes refer to a 2-functor simply as a functor, or, more informally, a map of 2-categories. A 2-functor between 2-groupoids is simply a 2-functor between the underlying 2categories.

In this paper, we encounter various kinds of "hom"s. To make it easier to keep track of these we follow certain conventions. First, the underlined ones are internal, namely they are 2-groupoids or 2-categories. The more curly they get, the more weakness is involved. E.g., Hom means the 2-category of strict 2-functors, strict 2 -transformations and modifications. $\underline{\mathcal{H O M}}$ then stands for the 2-category of weak 2-functors, weak transformations and modifications. Hom is used for simplicial mapping space in the category of simplicial sets.

For objects $A$ and $B$ in a category $\mathbf{C}$ with a notion of weak equivalence, we denote the set of morphisms in the homotopy category from $B$ to $A$, that is $\operatorname{Hom}_{\mathrm{Ho}(\mathbf{C})}(B, A)$, by $[B, A]_{\mathbf{C}}$.

The terms 'morphism', '1-morphism' and 'arrow' will be used synonymously. We use multiplicative notation for 1-morphisms of a 2-category, as opposed to the compositional notation (that is, $f g$ instead of $g \circ f$ ). We also do the same with the horizontal and vertical composition of 2-morphisms. To avoid confusion, we put our 2-morphisms inside square brackets when we are doing vertical compositions. This is all illustrated in the following example:

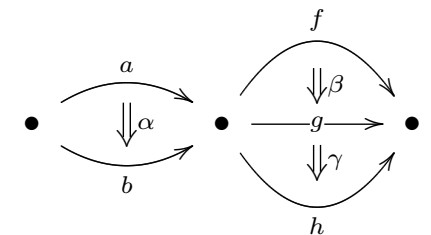

For example, the 2-morphism from af to $a g$ is denoted by $a \beta$, and the 2-morphism from af to $b h$ is given by

$$
[a \beta][a \gamma][\alpha h]=[a \beta][\alpha h][b \gamma]=[\alpha f][b \beta][b \gamma]=[\alpha \beta][b \gamma]=[a \beta][\alpha \gamma] .
$$

We use the German letters $\mathfrak{C}, \mathfrak{D}, \ldots$ for general 2 -categories and $\mathfrak{G}, \mathfrak{H}, \ldots$ for 2-groupoids. The upper case Latin letters $A, B, C, \ldots$ are used for objects in such 2-categories, lower case script letters $a, b, g, h, \ldots$ for 1-morphisms, and lower case Greek letters $\alpha, \beta, \ldots$ for 2-morphisms.

The symbol $\simeq$ is used for weak or homotopy equivalences, and $\cong$ is used for isomorphisms.

We denote by $\mathbf{2 G p d}$ the category of small 2-groupoids and strict functors, by $\mathbf{2 G}_{w}$ the category of small 2-groupoids and weak functors (Section 7), and by W2Gpd the category of small weak 2-groupoids and weak 2-functors (Section 9). Our notions of weak 2-functor and weak 2-category are slightly stronger than the standard notions in that we do not weaken the identities. The category of simplicial sets is denoted by SSet, and the category of small 2-categories by 2Cat. Various nerve functors that appear in this paper are all denoted by $N$. The Whitehead 
functor $W:$ SSet $\rightarrow \mathbf{2 G p d}$, to be introduced in Section 5 , is the left adjoint to $N: \mathbf{2 G p d} \rightarrow$ SSet.

By fiber product of 2-categories we mean strict fiber product. We will not encounter the homotopy fiber product of 2-categories in this paper.

For notation concerning crossed modules see $\S 3.2$.

\section{Quick review of 2-groups and crossed modules}

\subsection{Quick review of 2-groups}

We recall some basic facts about 2-groups and crossed modules. Our main references are [BaLa, $\mathbf{B a}, \mathbf{B r}, \mathbf{L o}, \mathbf{M o S e}, \mathbf{M c W h}, \mathbf{W h}]$. The reader is also encouraged to consult the works of the Bangor school (mostly available on their webpage [Ban]).

A 2-group $\mathfrak{G}$ is a group object in the category of groupoids. Alternatively, we can define a 2-group to be a groupoid object in the category of groups, or also, as a (strict) 2-category with one object in which all 1-morphisms and 2-morphisms are invertible (in the strict sense). We will try to adhere to the 'group in groupoids' point of view throughout the paper, but occasionally switching back and forth between different points of view is inevitable. Therefore, the reader will find it rewarding to master how the equivalence of these three point of views works.

A (strict) morphism $f: \mathfrak{G} \rightarrow \mathfrak{H}$ of 2 -groups is a map of groupoids that respects the group operation. If we view $\mathfrak{G}$ and $\mathfrak{H}$ as 2 -categories with one object, such $f$ is nothing but a strict 2 -functor. The category of 2 -groups is denoted by $\mathbf{2 G p}$.

To a 2-group $\mathfrak{G}$ we associate the groups $\pi_{1} \mathfrak{G}$ and $\pi_{2} \mathfrak{G}$ as follows. The group $\pi_{1} \mathfrak{G}$ is the set of isomorphism classes of objects of the groupoid $\mathfrak{G}$. The group structure on $\pi_{1} \mathfrak{G}$ is induced from the group operation of $\mathfrak{G}$. The group $\pi_{2} \mathfrak{G}$ is the group of automorphisms of the identity object $e \in \mathfrak{G}$. This is an abelian group. The groups $\pi_{1}$ and $\pi_{2}$ are functorial in $\mathfrak{G}$. A morphism $f: \mathfrak{G} \rightarrow \mathfrak{H}$ of 2-groups is called an equivalence if it induces isomorphisms on $\pi_{1}$ and $\pi_{2}$. The homotopy category of 2 -groups is the category obtained by inverting all the equivalences in $\mathbf{2} \mathbf{G p}$. We denote it by $\mathrm{Ho}(\mathbf{2} \mathbf{G p})$.

Caveat: an equivalence between 2-groups need not have an inverse. Also, two equivalent 2-groups may not be related by an equivalence, but only a zig-zag of equivalences.

\subsection{Quick review of crossed modules}

A crossed module $\mathfrak{G}=\left[\varphi: G_{2} \rightarrow G_{1}\right]$ is a pair of groups $G_{1}, G_{2}$, a group homomorphism $\varphi: G_{2} \rightarrow G_{1}$, and a (right) action of $G_{1}$ on $G_{2}$, denoted $-^{a}$, which lifts the conjugation action of $G_{1}$ on the image of $\varphi$ and descends the conjugation action of $G_{2}$ on itself. Before making this precise, let us introduce some (perhaps nonstandard) notation.

Notation. We usually denote the elements of $G_{2}$ by Greek letters and those of $G_{1}$ by lower case Roman letters. We sometimes suppress $\varphi$ from the notation and denote $\varphi(\alpha)$ by $\underline{\alpha}$. For elements $g$ and $a$ in a group $G$ we sometimes denote $a^{-1} g a$ by $g^{a}$. 
With the above notation, the two compatibility axioms of a crossed module can be written in the following way:

CM1. $\forall \alpha, \beta \in G_{2}, \beta^{\underline{\alpha}}=\beta^{\alpha}$;

CM2. $\forall \beta \in G_{2}, \forall a \in G_{1}, \underline{\beta}^{a}=\beta^{a}$.

The kernel of $\varphi$ is a central (in particular abelian) subgroup of $G_{2}$ and is denoted by $\pi_{2} \mathfrak{G}$. The image of $\varphi$ is a normal subgroup of $G_{1}$ whose cokernel is denoted by $\pi_{1} \mathfrak{G}$. A (strict) morphism $P: \mathfrak{H} \rightarrow \mathfrak{G}$ of crossed modules is a pair of group homomorphisms $p_{2}: H_{2} \rightarrow G_{2}$ and $p_{1}: H_{1} \rightarrow G_{1}$ which commute with the $\varphi$ maps and respect the actions. Such a morphism induces homomorphisms on $\pi_{1}$ and $\pi_{2}$. A morphism is called an equivalence if it induces isomorphisms on $\pi_{1}$ and $\pi_{2}$.

\subsection{Equivalence of 2-groups and crossed modules}

There is a natural pair of inverse equivalences between the category $\mathbf{2} \mathbf{G p}$ of 2-groups and the category CrossedMod of crossed modules. Furthermore, these functors preserve $\pi_{1}$ and $\pi_{2}$. They are constructed as follows. We only describe the effect of these functors on objects.

Functor from 2-groups to crossed modules. Let $\mathfrak{G}$ be a 2-group. Let $G_{1}$ be the group of objects of $\mathfrak{G}$, and $G_{2}$ the set of arrows emanating from the identity object $e$; the latter is also a group (namely, it is a subgroup of the group of arrows of $\mathfrak{G}$ ).

Define the map $\varphi: G_{2} \rightarrow G_{1}$ by sending $\alpha \in G_{2}$ to $t(\alpha)$.

The action of $G_{1}$ on $G_{2}$ is given by conjugation. That is, given $\alpha \in G_{2}$ and $g \in G_{1}$, the action is given by $g^{-1} \alpha g$. Here were are thinking of $g$ as an identity arrow and multiplication takes place in the group of arrows of of $\mathfrak{G}$. It is readily checked that $\left[\varphi: G_{2} \rightarrow G_{1}\right]$ is a crossed module.

Functor from crossed modules to 2-groups. Let $\left[\varphi: G_{2} \rightarrow G_{1}\right]$ be a crossed module. Consider the groupoid $\mathfrak{G}$ whose underlying set of objects is $G_{1}$ and whose set of arrows is $G_{1} \rtimes G_{2}$. The source and target maps are given by $s(g, \alpha)=g, t(g, \alpha)=$ $g \varphi(\alpha)$.

Recall that the group operation in $G_{1} \rtimes G_{2}$ is defined by $(g, \alpha)(h, \beta)=\left(g h, \alpha^{h} \beta\right)$. It is easy to see that $s, t$, and the inclusion map $G_{1} \hookrightarrow G_{1} \rtimes G_{2}$ are group homomorphisms. So $\mathfrak{G}$ is naturally a group object in the category of groupoids.

The above discussion shows that there is a pair of inverse functors inducing an equivalence between CrossedMod and 2Gp. These functors respect $\pi_{1}$ and $\pi_{2}$. Therefore, we have an equivalence

$$
\mathrm{Ho}(\text { CrossedMod }) \rightleftarrows \mathrm{Ho}(\mathbf{2 G p}) \text {. }
$$

This equivalence respects the functors $\pi_{1}$ and $\pi_{2}$.

\section{2-categories and 2-groupoids}

In this section we quickly go over some basic facts and constructions for 2categories, and fix some terminology. 
By a 2-category we mean a strict 2-category. A 2-groupoid is a 2-category in which every 1-morphism and every 2-morphism has an inverse (in the strict sense). Every category (respectively, groupoid) can be thought of as a 2-category (respectively, 2-groupoid) in which all 2-morphisms are identity.

A 2-functor between 2-categories means a strict 2-functor. We sometimes refer to a 2-functor simply by a functor, or a map of 2-categories. A 2-functor between 2 -groupoids is simply a 2 -functor between the underlying 2-categories.

\subsection{Strict transformations between 2-functors}

Let $\mathfrak{C}$ and $\mathfrak{D}$ be (strict) 2-categories, and let $P, Q: \mathfrak{D} \rightarrow \mathfrak{C}$ be (strict) 2-functors. By a 2-transformation $T: P \Rightarrow Q$ we mean an assignment of an arrow $t_{A}: P(A) \rightarrow$ $Q(A)$ in $\mathfrak{C}$ to every object $A$ in $\mathfrak{D}$ such that for every 2-morphism $\gamma: c \Rightarrow c^{\prime}$ in $\mathfrak{D}$ between two arrows $c$ and $c^{\prime}$ with common source and targets $A$ and $B$, we have $P(\gamma) t_{B}=t_{A} Q(\gamma)$. This can be depicted as the following commutative pillow:

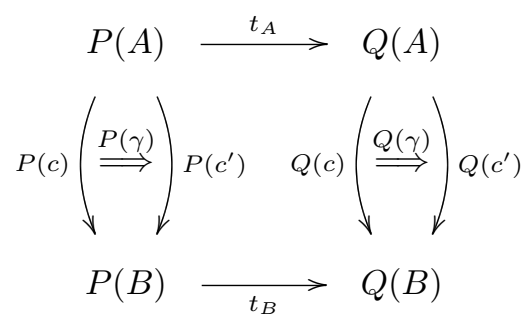

Alternatively, a 2-transformation $P \Rightarrow Q$ is the same as a 2-functor $\mathbf{I} \times \mathfrak{D} \rightarrow \mathfrak{C}$ whose restriction to $\{0\} \times \mathfrak{D}$ is $P$ and whose restriction to $\{1\} \times \mathfrak{D}$ is $Q$. Here $\mathbf{I}$ stands for the category $\{0 \rightarrow 1\}$. We sometimes refer to a 2-transformation simply by a transformation, if there is no chance of confusion.

A transformation between two 2-transformations $S, T$, called a modification, is a rule assigning to each object $A \in \mathfrak{D}$ a 2-morphism $\mu_{A}: s_{A} \Rightarrow t_{A}$ in $\mathfrak{C}$, such that for every arrow $c: A \rightarrow B$ in $\mathfrak{D}$, we have $P(c) \mu_{B}=\mu_{A} Q(c)$. This can be depicted as the following commutative pillow:

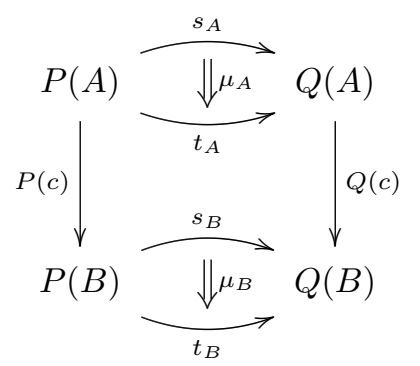

Alternatively, a modification $S \Rightarrow T$ is the same as a 2 -functor $\mathbb{I} \times \mathfrak{D} \rightarrow \mathfrak{C}$ whose restriction to the subcategory $(s, \mathrm{id}): \mathbf{I} \times \mathfrak{D} \hookrightarrow \mathbb{I} \times \mathfrak{D}$ corresponds to the transformation $S$, and whose restriction to the subcategory $(t, \mathrm{id}): \mathbf{I} \times \mathfrak{D} \hookrightarrow \mathbb{I} \times \mathfrak{D}$ corresponds 
to the transformation $T$. Here $\mathbb{I}$ stands for the 2-category

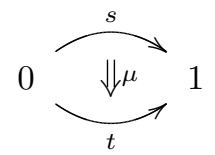

Definition 4.1. Given 2-categories $\mathfrak{C}$ and $\mathfrak{D}$, we define $\operatorname{Hom}(\mathfrak{D}, \mathfrak{C})$ to be the 2category whose objects are 2-functors from $\mathfrak{D}$ to $\mathfrak{C}$, whose 1 -morphisms are 2transformations between 2-functors, and whose 2-morphisms are modifications.

The following exponential law is easy to prove.

Lemma 4.2. Let $\mathfrak{C}, \mathfrak{D}$ and $\mathfrak{E}$ be 2-categories, then we have a natural isomorphism of 2-categories

$$
\underline{\operatorname{Hom}}(\mathfrak{E} \times \mathfrak{D}, \mathfrak{C}) \cong \underline{\operatorname{Hom}}(\mathfrak{E}, \underline{\operatorname{Hom}}(\mathfrak{D}, \mathfrak{C}))
$$

With this, 2Cat is enriched in 2-categories. When $\mathfrak{C}$ is a 2-groupoid, $\underline{\operatorname{Hom}}(\mathfrak{D}, \mathfrak{C})$ is also a 2-groupoid. So, $\mathbf{2 G}$ pd is naturally enriched in 2-groupoids. In other words, 2Cat and 2Gpd are closed monoidal categories, the product being cartesian product.

\subsection{Weak transformations between 2-functors}

There is also a notion of weak 2-transformation between (strict) 2-functors, and modifications between them. We recall the definitions.

A weak transformation between two 2-functors $P$ and $Q$ is like a strict transformation, except that to a morphism $c: A \rightarrow B$ in $\mathfrak{D}$ we assign a 2-cell

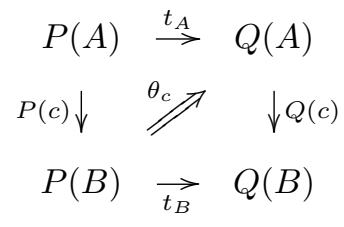

We require that $\theta_{\mathrm{id}}=\mathrm{id}$, and that $\theta_{h}$ satisfy the obvious compatibility conditions with respect to 2 -morphisms and composition of morphisms. There are two types of conditions here. One is the same as the commutative pillow of page 81 , in which the top and bottom squares are now decorated with $\theta_{c}$ and $\theta_{c^{\prime}}$, respectively. (Just to make sure the reader visualizes the pillow correctly, the top square of the pillow is exactly the 2-commutative square of the previous paragraph.) The second compatibility condition says that for every two composable morphisms $a$ and $b$, the corresponding squares compose. By abuse of notation, this can be written as $\theta_{a} \theta_{b}=\theta_{a b}$.

Modifications between two weak 2-transformations are defined in the same way as they were defined for strict 2-transformations ( $\$ 4.1)$. 
Definition 4.3. Given 2-categories $\mathfrak{C}$ and $\mathfrak{D}$, we define $\underline{\mathcal{H} o m}(\mathfrak{D}, \mathfrak{C})$ to be the 2category whose objects are 2 -functors from $\mathfrak{D}$ to $\mathfrak{C}$, whose 1 -morphisms are weak 2 -transformations between 2 -functors, and whose 2 -morphisms are modifications. ${ }^{1}$

Of course, the exponential law of Lemma 4.2 will not work with $\underline{\mathcal{H} o m}$ unless we change our product accordingly. It turns out that this can be done, namely by replacing the usual product with the Gray tensor product. For a nice description of Gray tensor product see [La02], last paragraph of Section 2.

In the following, $\otimes$ stands for the Gray tensor product.

Lemma $4.4([\mathbf{G r}]$, page 73, Theorem I,4.9). Let $\mathfrak{C}, \mathfrak{D}$ and $\mathfrak{E}$ be 2-categories, then we have a natural isomorphism of 2-categories

$$
\underline{\mathcal{H o m}}(\mathfrak{E} \otimes \mathfrak{D}, \mathfrak{C}) \cong \underline{\mathcal{H} o m}(\mathfrak{E}, \underline{\mathcal{H} o m}(\mathfrak{D}, \mathfrak{C})) .
$$

This gives us another enrichment of $\mathbf{2 C a t}$ over itself. When $\mathfrak{C}$ is a 2-groupoid, then $\operatorname{Hom}(\mathfrak{D}, \mathfrak{C})$ is also a 2-groupoid. So, similarly, we have a new enrichment of $\mathbf{2 G p d}$ over itself. Therefore, each of $\mathbf{2 G} \mathbf{p d}$ and $\mathbf{2 C a t}$ admits two different closed monoidal structures, one with $\underline{\text { Hom }}$ and cartesian product, the other with $\underline{\mathcal{H} o m}$ and Gray tensor product.

\subsection{Nerve of a 2-category}

We review the nerve construction for 2-categories, and recall its basic properties [MoSe].

Let $\mathfrak{G}$ be a 2-category. We define the nerve of $\mathfrak{G}$, denoted by $N \mathfrak{G}$, to be the simplicial set defined as follows. The set of 0 -simplices of $N \mathfrak{G}$ is the set of objects of $\mathfrak{G}$. The 1 -simplices are the morphisms in $\mathfrak{G}$. The 2 -simplices are diagrams of the form

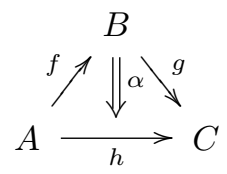

where $\alpha: f g \Rightarrow h$ is a 2-morphism. The 3 -simplices of $N \mathfrak{G}$ are commutative tetrahedra of the form

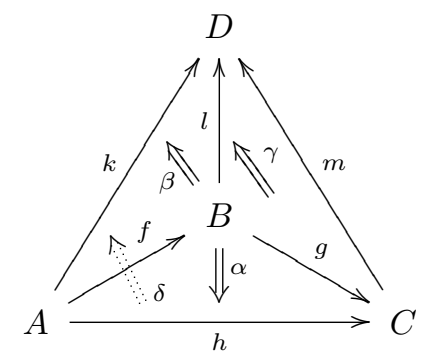

Commutativity of the above tetrahedron means $[f \gamma][\beta]=[\alpha m][\delta]$. That is, the

\footnotetext{
${ }^{1}$ In $[\mathbf{G r}]$, this 2-category is denoted by Fun.
} 
following square of transformations is commutative:

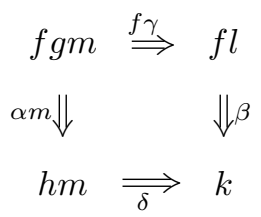

For $n \geqslant 3$, an $n$-simplex of $N \mathfrak{G}$ is an $n$-simplex such that each of its sub 3 -simplices is a commutative tetrahedron as described above. In other words, $N \mathfrak{G}$ is the coskeleton of the 3 -truncated simplicial set $\left\{N \mathfrak{G}_{0}, N \mathfrak{G}_{1}, N \mathfrak{G}_{2}, N \mathfrak{G}_{3}\right\}$ defined above.

The nerve gives us a functor $N: \mathbf{2 C a t} \rightarrow$ SSet, where SSet is the the category of simplicial sets.

\section{Closed model structure on 2Gpd}

We quickly review the Moerdijk-Svensson closed model structure on the category of 2-groupoids. The main reference is [MoSe]. A generalization of this model structure to the case of crossed-complexes can be found in [BrGo].

Definition 5.1. Let $\mathfrak{H}$ and $\mathfrak{G}$ be 2-groupoids, and $P: \mathfrak{H} \rightarrow \mathfrak{G}$ a functor between them. We say that $P$ is a fibration if it satisfies the following properties:

F1. For every arrow $a: A_{0} \rightarrow A_{1}$ in $\mathfrak{G}$, and every object $B_{1}$ in $\mathfrak{H}$ such that $P\left(B_{1}\right)=$ $A_{1}$, there is an object $B_{0}$ in $\mathfrak{H}$ and an arrow $b: B_{0} \rightarrow B_{1}$ such that $P(b)=a$.

F2. For every 2-morphism $\alpha: a_{0} \Rightarrow a_{1}$ in $\mathfrak{G}$ and every arrow $b_{1}$ in $\mathfrak{H}$ such that $P\left(b_{1}\right)=a_{1}$, there is an arrow $b_{0}$ in $\mathfrak{H}$ and a 2-morphism $\beta: b_{0} \Rightarrow b_{1}$ such that $P(\beta)=\alpha$.

Definition 5.2. Let $\mathfrak{G}$ be a 2 -groupoid, and $A$ an object in $\mathfrak{G}$. We define the following.

- $\pi_{0} \mathfrak{G}$ is the set of isomorphism classes of objects in $\mathfrak{G}$.

- $\pi_{1}(\mathfrak{G}, A)$ is the group of 2-isomorphism classes of arrows from $A$ to itself. The fundamental groupoid $\Pi_{1} \mathfrak{G}$ is the groupoid obtained by taking the groupoid of all 1-morphisms of $\mathfrak{G}$ and identifying 2-isomorphic 1-morphisms.

- $\pi_{2}(\mathfrak{G}, A)$ is the group of 2-automorphisms of the identity arrow $1_{A}: A \rightarrow A$.

These invariants are functorial with respect to 2 -functors. A map $\mathfrak{H} \rightarrow \mathfrak{G}$ is called a (weak) equivalence of 2-groupoids if it induces bijections on $\pi_{0}, \pi_{1}$ and $\pi_{2}$ for every choice of basepoint. We usually drop the adjective 'weak' when we talk about weak equivalences of 2-groupoids, groupoids, 2-groups, or crossed modules.

We remark that a 2 -group in the sense of $\S 3.1$ is precisely a 2 -groupoid with one object. This identifies $\mathbf{2 G p}$ as a full subcategory of $\mathbf{2 G} \mathbf{p d}$. Under this identification, the notions of $\pi_{1}, \pi_{2}$, and weak equivalence introduced in $\S 3.1$ coincide with the ones given above.

Having defined fibrations and weak equivalences between 2-groupoids, we define cofibrations using the left lifting property. 
Theorem 5.3 ([MoSe], Theorem 1.2). With weak equivalences, fibrations and cofibrations defined as above, the category of 2-groupoids is a closed model category.

In this model structure, every object is fibrant. Cofibrant objects are a bit trickier, but, morally, they should be thought of as some sort of free objects. A more explicit description of cofibrations in the Moerdijk-Svensson model structure is given in the Remark on page 194 of [MoSe].

The nerve functor is a bridge between the homotopy theory of 2-groupoids and the homotopy theory of simplicial sets. To justify this statement, we quote the following from [MoSe].

Proposition 5.4 (see [MoSe], Proposition 2.1).

i. The functor $N: \mathbf{2 C a t} \rightarrow$ SSet sends transformations between 2-functors to simplicial homotopies, is faithful and preserves fiber products. (We will see in Section 7 that, in contrast to the case of ordinary categories, $N$ is not full.)

ii. The functor $N: \mathbf{2 G} \mathbf{p d} \rightarrow \mathbf{S S e t}$ sends a fibration between 2-groupoids (Definition 5.1) to a Kan fibration. Conversely, if a map $P$ of 2-groupoids is sent to a Kan fibration, then $P$ itself is a fibration. The nerve of every 2-groupoid is a Kan complex.

iii. For every (pointed) 2-groupoid $\mathfrak{G}$ we have $\pi_{i}(\mathfrak{G}) \cong \pi_{i}(N \mathfrak{G}), i=0,1,2$.

iv. A map $f: \mathfrak{H} \rightarrow \mathfrak{G}$ of 2 -groupoids is an equivalence if and only if $N f: N \mathfrak{H} \rightarrow$ $N \mathfrak{G}$ is a weak equivalence of simplicial sets.

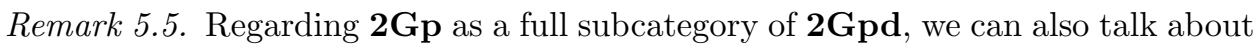
nerves of 2-groups. This is a functor $N: \mathbf{2} \mathbf{G p} \rightarrow \mathbf{S S e t}_{*}$, where $\mathbf{S S e t}_{*}$ is the category of pointed simplicial sets. The above proposition remains valid if we replace 2groupoids by 2-groups and $\mathbf{S S e t}$ by $\mathbf{S S e t}_{*}$ throughout.

The functor $N: \mathbf{2 G} \mathbf{p d} \rightarrow$ SSet has a left adjoint $W:$ SSet $\rightarrow \mathbf{2 G}$ pd, called the Whitehead 2-groupoid, which is defined as follows (see [MoSe] page 190, Example 2 ). Let $X$ be a simplicial set. The underlying groupoid of $W(X)$ is $\Pi_{1}\left(\left|X^{(1)}\right|,\left|X^{(0)}\right|\right)$, where $\left|X^{(i)}\right|$ stands for the geometric realization of the $i^{\text {th }}$ skeleton of $X$, and $\Pi_{1}$ stands for fundamental groupoid. A 2-morphism in $W(X)$ is the equivalence class of a continuous map $\alpha: I \times I \rightarrow|X|$ such that $\alpha(\{0,1\} \times I) \subseteq\left|X^{(0)}\right|$ and $\alpha(I \times\{0,1\}) \subseteq\left|X^{(1)}\right|$. Two such maps $\alpha$ and $\beta$ are equivalent if there is homotopy $H:(I \times I) \times I \rightarrow|X|$ between them such that $H((\{0,1\} \times I) \times I) \subseteq\left|X^{(0)}\right|$ and $H((I \times\{0,1\}) \times I) \subseteq\left|X^{(1)}\right|$.

It is easy to see that $W$ preserves homotopy groups. In particular, it sends weak equivalences of simplicial sets to equivalences of 2-groupoids. Much less obvious is the following

Theorem 5.6 ([MoSe], Section 2). The pair

$$
W: \text { SSet } \rightleftharpoons 2 \text { Gpd }: N
$$

is a Quillen pair. It satisfies the following properties:

i. Each adjoint preserves weak equivalences. 
ii. For every 2-groupoid $\mathfrak{G}$, the counit of adjunction $W N(\mathfrak{G}) \rightarrow \mathfrak{G}$ is a weak equivalence.

iii. For every simplicial set $X$ such that $\pi_{i} X=0, i \geqslant 3$, the unit of adjunction $X \rightarrow N W(X)$ is a weak equivalence.

In particular, the functor $N: \mathrm{Ho}(\mathbf{2 G p d}) \rightarrow \mathrm{Ho}($ SSet $)$ induces an equivalence of categories between $\mathrm{Ho}(\mathbf{2} \mathbf{G p d})$ and the category of homotopy 2-types. (The latter is defined to be the full subcategory of $\mathrm{Ho}$ (SSet) consisting of all $X$ such that $\pi_{i} X=0$, $i \geqslant 3$.)

Remark 5.7. The pointed version of the above theorem is also true. The proof is just a minor modification of the proof of the above theorem.

It is also well-known that the geometric realization functor $|-|: \mathbf{S S e t}_{*} \rightarrow \mathbf{T o p}_{*}$ induces an equivalence of of homotopy categories. So we have the following

Corollary 5.8. The functor $|N(-)|: \mathrm{Ho}(\mathbf{2} \mathbf{G p}) \rightarrow \mathrm{Ho}\left(\mathbf{T o p}_{*}\right)$ induces an equivalence between $\mathrm{Ho}(\mathbf{2} \mathbf{G p})$ and the full subcategory of $\mathrm{Ho}\left(\mathbf{T o p}_{*}\right)$ consisting of connected pointed homotopy 2-types.

\subsection{Monoidal closed model structure on $2 \mathrm{Gpd}$}

We saw in Section 5 that $\mathbf{2 G p d}$ admits a closed model structure. We also know from $\S 4.1$ and $\S 4.2$ that $\mathbf{2 G p d}$ can be made into a monoidal category in two different ways. Namely, there are two reasonable ways of enriching the hom-sets to 2-groupoids. In many applications, it is desirable to have simplicially enriched homsets. In other words, one would like to have a simplicial structure in the sense of ([GoJa], § II.2, Definition 2.1). Furthermore, to have a full-fledged homotopy theory one also requires a compatibility between the simplicial structure and the closed model structure. This compatibility condition is neatly encoded in Quillen's SM7, ([GoJa $], \S$ II.3, Definition 3.1).

We have two candidates for simplicially enriching $\mathbf{2 G p d}$. We could either take $N \underline{\operatorname{Hom}}(\mathfrak{H}, \mathfrak{G})$, or $N \underline{\mathcal{H} o m}(\mathfrak{H}, \mathfrak{G})$. It can be shown that 2Gpd does become a simplicial category with $N \underline{\operatorname{Hom}}(\mathfrak{H}, \mathfrak{G})$. However, SM7 will not be satisfied, because it is easy to produce examples where the (derived) mapping spaces do not have the right homotopy type (or one can directly verify that SM7 fails). At first look, $N \underline{\mathcal{H} o m}(\mathfrak{H}, \mathfrak{G})$ seems to be the correct alternative, because in this setting the (derived) mapping spaces can be shown to have the right homotopy type. However this attempt fails even more miserably, as this simplicial enrichment is not even well-defined: given three groupoids $\mathfrak{G}, \mathfrak{H}$ and $\mathfrak{K}$, the composition map

$$
N \underline{\mathcal{H} \text { om }}(\mathfrak{K}, \mathfrak{H}) \times N \underline{\mathcal{H} o m}(\mathfrak{H}, \mathfrak{G}) \rightarrow N \underline{\mathcal{H} o m}(\mathfrak{K}, \mathfrak{G})
$$

can not even be defined! This is because if we have an arrow in $\underline{\mathcal{H} o m}(\mathfrak{K}, \mathfrak{H})$, namely a weak 2-transformation between the 2 -functors $P_{1}, Q_{1}: \mathfrak{K} \rightarrow \mathfrak{H}$, and an arrow in $\underline{\mathcal{H} o m}(\mathfrak{H}, \mathfrak{G})$, namely a weak 2 -transformation between the 2 -functors $P_{2}, Q_{2}: \mathfrak{H} \rightarrow$ $\mathfrak{G}$, then it is not possible to define a canonical weak transformation between the 2 -functors $P_{2} \circ P_{1}, Q_{2} \circ Q_{1}: \mathfrak{K} \rightarrow \mathfrak{G}$ out of these.

In $[\mathbf{L a 0 4}]$ it is proven that the monoidal structure on $\mathbf{2} \mathbf{G p d}$ defined via $\mathcal{H o m}$ and Gray tensor product is compatible with the Moerdijk-Svensson model structure. 
Namely, it equips $\mathbf{2 G p d}$ with the structure of a monoidal closed model category in the sense of $([\mathbf{L a 0 4}], \S 7)$. The nerve functor $N: \mathbf{2 G} \mathbf{\text { pd }} \rightarrow$ SSet, however, does not respect the monoidal structure.

Nevertheless, with some extra work, one can prove that $N$ respects derived mapping spaces (Proposition 7.9).

Definition 5.9. Let $\mathbf{C}$ be a monoidal closed model category, with internal homobjects denoted by $\mathcal{H o m}$. Let $A$ and $B$ be objects in $\mathbf{C}$. The derived hom-object $\underline{\mathcal{R H o m}}(B, A)$ is defined to be $\underline{\mathcal{H} o m}(\bar{B}, \bar{A})$, where $\bar{A}$ is a fibrant replacement for $A$ and $\bar{B}$ is a cofibrant replacement for $B$.

The derived hom-object should be thought of as the monoidal enrichment of $[B, A]_{\mathbf{C}}$, in the sense that $\pi_{0} \mathcal{R H o m}(B, A) \cong[B, A]_{\mathbf{C}}$. The reason for making such fibrant-cofibrant replacements is that $\underline{\mathcal{H} o m}(B, A)$ is not a priori homotopy invariant, in the sense that its weak homotopy type may change if we replace $A$ or $B$ by a weakly equivalent object. However, $\underline{\mathcal{R H o m}}(B, A)$ as defined above is homotopy invariant. Of course, there are some choices involved in the definition of $\underline{\mathcal{R H o m}}(B, A)$, but the weak homotopy type of $\mathcal{R} \mathcal{H o m}(B, A)$ is well-defined.

In Section 7 we give an explicit model for $\underline{\mathcal{R H o m}}(\mathfrak{H}, \mathfrak{G})$ using weak 2-functors; see Proposition 7.8.

\section{The pointed category $2 \mathrm{Gpd}_{*}$ and application to crossed modules}

In this section we discuss pointed versions of the results of the previous section. The interest in the pointed version lies in the fact that understanding the homotopy theory of pointed 2 -groupoids $\mathbf{2} \mathbf{G} \mathbf{p d} \mathbf{d}_{*}$ is what we need in studying $\mathbf{2} \mathbf{G p}$ and CrossedMod.

We define the category $\mathbf{2} \mathbf{C a t} *$ of pointed 2-categories as follows. A pointed 2category is a 2-category with a chosen base point (i.e. object) which, by abuse of notation, we denote by $*$. In $\mathbf{2} \mathbf{C a t}_{*}$ a 2 -functor $P: \mathfrak{D} \rightarrow \mathfrak{C}$ is required to preserve the base points, and a transformation $T: P \Rightarrow Q$ between such functors is required to satisfy the condition $\theta_{*}=\mathrm{id}_{*}$ (similarly, for modifications we require $\mu_{*}$ to be the identity). The pointed hom-2-category between two pointed 2-categories $\mathfrak{D}$ and $\mathfrak{C}$ is denoted by $\underline{\mathcal{H o m}}_{*}(\mathfrak{D}, \mathfrak{C})$.

The same definitions apply to the category $\mathbf{2} \mathbf{G p d}_{*}$ of 2 -groupoids. There is a pointed version of the Moerdijk-Svensson closed model structure, and pointed versions of Proposition 5.4 and Theorem 5.6 are valid.

The following proposition follows from the pointed version of Theorem 5.6.

Proposition 6.1. The functor $N: \mathrm{Ho}(\mathbf{2 G p}) \rightarrow \mathrm{Ho}\left(\mathbf{S S e t}_{*}\right)$ induces an equivalence between $\mathrm{Ho}(\mathbf{2} \mathbf{G p})$ and the full subcategory of $\mathrm{Ho}\left(\mathbf{S S e t}_{*}\right)$ consisting of connected pointed homotopy 2-types.

We point out that every pointed 2 -groupoid is fibrant in $\mathbf{2} \mathbf{G p d} \mathbf{d}_{*}$. It is also easy to check that a pointed 2-groupoid is cofibrant in $\mathbf{2} \mathbf{G p d}_{*}$ if and only if it is cofibrant in $\mathbf{2 G p d}$. 


\subsection{Translation to the language of crossed modules}

We can now apply the homotopy theory developed above for $\mathbf{2} \mathbf{G p d}_{*}$ to study 2-groups. This is because $\mathbf{2} \mathbf{G}$ p naturally embeds as a full subcategory of $\mathbf{2} \mathbf{G}_{\mathbf{p d}}$, by viewing a 2-group as a 2-groupoid with one object. On the other hand, 2-groups are the same as crossed modules (see §3.3), so it is interesting to translate the homotopical structure of $\mathbf{2} \mathbf{G} \mathbf{p d}$ * through $\mathbf{2} \mathbf{G p}$ to the language of crossed modules.

We start with the notions of transformation and pointed transformation. Let $\mathfrak{G}=\left[\varphi: G_{2} \rightarrow G_{1}\right]$ and $\mathfrak{H}=\left[\psi: H_{2} \rightarrow H_{1}\right]$ be crossed modules, and let $P, Q: \mathfrak{H} \rightarrow \mathfrak{G}$ be morphisms between them. Then a transformation $T: P \Rightarrow Q$ is given by a pair $(a, \theta)$ where $a \in G_{1}$ and $\theta: H_{1} \rightarrow G_{2}$ is a crossed homomorphism for the induced action, via $p_{1}^{a}$, of $H_{1}$ on $G_{2}$ (that is, $\left.\theta\left(h h^{\prime}\right)=\theta(h)^{p_{1}\left(h^{\prime}\right)^{a}} \theta\left(h^{\prime}\right)\right)$. These data should satisfy the following axioms:

T1. $p_{1}(h)^{a} \theta(h)=q_{1}(h)$, for every $h \in H_{1}$;

T2. $p_{2}(\beta)^{a} \overline{\theta(\underline{\beta})}=q_{2}(\beta)$, for every $\beta \in H_{2}$.

A transformation $T$ is pointed if and only if $a=1$. Between two pointed transformations there is no non-trivial pointed modification.

Definition 6.2. We say a morphism $\mathfrak{H} \rightarrow \mathfrak{G}$ of crossed modules is a fibration (respectively, cofibration) if the induced morphism on the associated 2-groups is so (in the sense of Moerdijk-Svensson).

Proposition 6.3. A map $\left(f_{2}, f_{1}\right):\left[H_{2} \rightarrow H_{1}\right] \rightarrow\left[G_{2} \rightarrow G_{1}\right]$ of crossed modules is a fibration if $f_{2}$ and $f_{1}$ are both surjective. It is a trivial fibration if, furthermore, the map $H_{2} \rightarrow H_{1} \times{ }_{G_{1}} G_{2}$ is an isomorphism.

Note that every crossed module is fibrant.

Proposition 6.4. A crossed module $\left[G_{2} \rightarrow G_{1}\right]$ is cofibrant if and only if $G_{1}$ is a free group.

Proof. This follows immediately from the Remark on page 194 of [MoSe], but we give a direct proof. A 2-group $\mathfrak{G}$ is cofibrant in the Moerdijk-Svensson structure if and only if every trivial fibration $\mathfrak{H} \rightarrow \mathfrak{G}$, where $\mathfrak{H}$ is a 2-groupoid, admits a section. But we can obviously restrict ourselves to 2-groups $\mathfrak{H}$. So we can work entirely within crossed modules, and use the crossed module version of trivial fibrations as in Proposition 6.3.

Assume $G_{1}$ is free. Let $\left(f_{2}, f_{1}\right):\left[H_{2} \rightarrow H_{1}\right] \rightarrow\left[G_{2} \rightarrow G_{1}\right]$ be a trivial fibration. Since $G_{1}$ is free and $f_{1}$ is surjective, there is a section $s_{1}: G_{1} \rightarrow H_{1}$. Using the fact that $H_{2} \cong H_{1} \times{ }_{G_{1}} G_{2}$, we also get a natural section $s_{2}: G_{2} \rightarrow H_{2}$ for the projection $H_{1} \times G_{1} G_{2} \rightarrow G_{2}$, namely, $s_{2}(\alpha)=\left(s_{1}(\underline{\alpha}), \alpha\right)$. It is easy to see that $\left(s_{2}, s_{1}\right):\left[G_{2} \rightarrow\right.$ $\left.G_{1}\right] \rightarrow\left[H_{2} \rightarrow H_{1}\right]$ is a map of crossed modules.

To prove the converse, choose a free group $F_{1}$ and a surjection $f_{1}: F_{1} \rightarrow G_{1}$. Form the pullback crossed module $\left[F_{2} \rightarrow F_{1}\right]$ by setting $F_{2}=F_{1} \times_{G_{1}} G_{2}$. Then we have a trivial fibration $\left[F_{2} \rightarrow F_{1}\right] \rightarrow\left[G_{2} \rightarrow G_{1}\right]$. By assumption, this has a section, so in particular we get a section $s_{1}: G_{1} \rightarrow F_{1}$ which embeds $G_{1}$ as a subgroup of $F_{1}$. It follows from Nielsen's theorem that $G_{1}$ is free. 
Remark 6.5. Let $G$ be a group and $H$ a subgroup. Let us say that $G$ is a free extension of $H$ if for every surjection $p: K \rightarrow G$, every partial section $s: H \rightarrow K$ to $p$ can be extended to $G$. For instance, if $H$ is the trivial group, this is equivalent to $G$ being free. Proposition 6.3 can be generalized by saying that $\left(f_{2}, f_{1}\right):\left[H_{2} \rightarrow\right.$ $\left.H_{1}\right] \rightarrow\left[G_{2} \rightarrow G_{1}\right]$ is a cofibration if and only if $f_{1}: H_{1} \rightarrow G_{1}$ is injective and $G_{1}$ is a free extension of $f_{1}\left(H_{1}\right)$.

Using the above proposition, we can give a simple recipe for cofibrant replacement of a crossed module. Let $\mathfrak{G}=\left[G_{2} \rightarrow G_{1}\right]$ be an arbitrary crossed module. Let $F_{1} \rightarrow$ $G_{1}$ be a surjective map from a free group $F_{1}$, and set $F_{2}:=F_{1} \times_{G_{1}} G_{2}$. Consider the crossed module $\mathfrak{F}=\left[F_{2} \rightarrow F_{1}\right]$. Then $\mathfrak{F}$ is cofibrant, and the natural map $\mathfrak{F} \rightarrow \mathfrak{G}$ is a trivial fibration (Proposition 6.3). In other words, $\mathfrak{F} \rightarrow \mathfrak{G}$ is a cofibrant replacement for $\mathfrak{G}$.

\section{Weak 2-functors between 2-categories}

In contrast to the case of ordinary groupoids, the nerve functor $N: \mathbf{2} \mathbf{G p d} \rightarrow$ SSet fails to be full. In order to make it full, one can cheat and define a weak 2-functor $\mathfrak{H} \rightarrow \mathfrak{G}$ to be a simplicial map $N \mathfrak{H} \rightarrow N \mathfrak{G}$ ! Translating this back to the language of 2-categories, we arrive at the following definition.

Definition 7.1. Let $\mathfrak{H}$ and $\mathfrak{G}$ be 2-categories. A weak 2-functor $F: \mathfrak{H} \rightarrow \mathfrak{G}$ is defined the same way as a strict 2-functor, except that for every pair of composable arrows $a, b \in \mathfrak{H}$ we require that instead of the equality $F(a b)=F(a) F(b)$, we are given a 2-morphism $\varepsilon_{a, b}: F(a) F(b) \Rightarrow F(a b)$ which is natural in $a$ and $b$. For every three composable arrows $a, b$ and $c$, we require that the following diagram commutes:

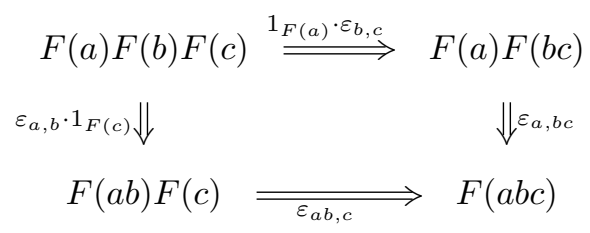

Remark 7.2.

i. The naturalness assumption on $\varepsilon_{a, b}$ in particular implies the following. Suppose we are given 2-morphisms as in the diagram

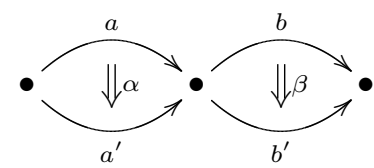

Then, we require that after applying $F$ the diagram remains commutative. This means

$$
\left[\varepsilon_{a, b}\right][F(\alpha, \beta)]=[F(\alpha) F(\beta)]\left[\varepsilon_{a^{\prime}, b^{\prime}}\right],
$$

as 2-morphisms from $F(a) F(b)$ to $F\left(a^{\prime} b^{\prime}\right)$. 
ii. Our definition of weak is slightly stronger than the usual one in that we are assuming that $F$ preserves the identity 1-morphisms strictly. We do not, however, require that $F\left(a^{-1}\right)=F(a)^{-1}$.

Considering 2-groups as 2-categories with one object, we obtain the notion of a weak map of 2 -groups. The translation of this definition in the language of crossed modules is given in Section 8.

We denote the category of 2-categories (respectively, 2-groupoids, 2-groups) with weak morphisms by $\mathbf{2} \mathbf{C a t}_{\mathrm{w}}$ (respectively, $\mathbf{2} \mathbf{G p d}_{\mathrm{w}}, \mathbf{2} \mathbf{G p}_{\mathrm{w}}$ ). Notice that each of these categories contains the corresponding strict category as a subcategory.

\subsection{Transformations between weak functors}

Let $\mathfrak{D}$ and $\mathfrak{C}$ be 2 -categories. Weak 2 -functors from $\mathfrak{D}$ to $\mathfrak{C}$ form a 2-category. The 1-morphism in this 2-category are transformations $T: P \Rightarrow Q$. Recall (§4.2) that a transformation between strict functors $P$ and $Q$ is a rule which assigns to every morphism $c: A \rightarrow B$ in $\mathfrak{D}$ a 2-cell in $\mathfrak{C}$ of the form

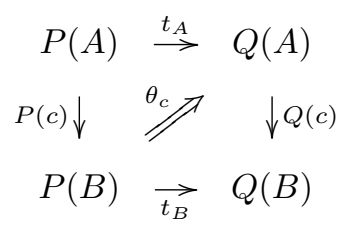

In case of weak 2 -functors $P$ and $Q$, we require the same conditions as in $\S 4.2$, except that the second compatibility condition " $\theta_{a} \theta_{b}=\theta_{a b}$ ", should be modified as follows. For every two composable morphisms $a$ and $b$, the prism (of the form $\left.\Delta^{1} \times \Delta^{2}\right)$ with three square faces $\theta_{a}, \theta_{b}$ and $\theta_{a b}$ and two triangular faces $\epsilon_{a, b}^{P}$ and $\epsilon_{a, b}^{Q}$ is commutative.

We can also talk about modification between two weak 2-transformations between weak 2-functors. The definition is identical to the one in the case of strict 2-functors $(\S 4.1)$.

Definition 7.3. Given 2-categories $\mathfrak{C}$ and $\mathfrak{D}$, we define $\underline{\mathcal{H O M}}(\mathfrak{D}, \mathfrak{C})$ to be the 2category whose objects are weak 2 -functors from $\mathfrak{D}$ to $\mathfrak{C}$, whose 1 -morphisms are weak 2 -transformations between 2 -functors, and whose 2 -morphisms are modifications.

Observe that $\underline{\mathcal{H O M}}(\mathfrak{D}, \mathfrak{C})$ contains $\underline{\mathcal{H} o m}(\mathfrak{D}, \mathfrak{C})$ as a full sub-2-category. In the case where $\mathfrak{C}$ is a 2 -groupoid, $\mathcal{H O M}(\mathfrak{D}, \mathfrak{C})$ is also a 2-groupoid.

\subsection{Nerves of weak functors}

As pointed out at the beginning of this section, our definition of a weak functor between two 2-categories was formulated by translating the simplicial identities that have to be satisfied by a simplicial map on the corresponding nerves. Having taken this for granted, the following is immediate. 
Proposition 7.4. The nerve functors extend to fully faithful functors

$$
N: \mathbf{2 C a t}_{w} \rightarrow \text { SSet, } N: \mathbf{2} \mathbf{G p d}_{w} \rightarrow \mathbf{S S e t} \text {, and } N: \mathbf{2 G}_{w} \rightarrow \mathbf{S S e t}_{*} .
$$

Remark 7.5. Using the notion of weak functor between 2-categories, we can give an alternative view to the nerve of a 2-category $\mathfrak{G}$. Let $\mathbf{I}_{n}$ be the ordered set $\{0,1, \cdots, n\}$, with the usual ordering, viewed as a category (hence, also a 2-category). Then $N \mathfrak{G}_{n}$ is in natural bijection with the set of weak functors $\mathbf{I}_{n} \rightarrow \mathfrak{G}$. This also explains why $N$ is functorial and preserves fiber products.

Lemma 7.6. A weak map $P: \mathfrak{H} \rightarrow \mathfrak{G}$ of 2-groupoids induces group homomorphisms $\pi_{i} P: \pi_{i} \mathfrak{H} \rightarrow \pi_{i} \mathfrak{G}, i \leqslant 2$, for every choice of base points. In particular, we can talk about a weak map $P$ being an equivalence of 2-groupoids. (Compare Definition 5.2.) Furthermore, $P: \mathfrak{H} \rightarrow \mathfrak{G}$ is an equivalence of 2-groupoids if and only if $N P: N \mathfrak{H} \rightarrow$ $N \mathfrak{G}$ is a weak equivalence of simplicial sets.

Proof. Straightforward.

The next result is that $\underline{\mathcal{H O M}}(\mathfrak{H}, \mathfrak{G})$ can be used to compute derived hom-2groupoids without the need to take a cofibrant replacement for $\mathfrak{H}$. To prove this fact, first we prove a lemma.

Lemma 7.7. Let $P, Q: \mathfrak{H} \rightarrow \mathfrak{G}$ be weak 2-functors between 2-groupoids. Then $P$ and $Q$, viewed as objects in the 2-groupoid $\underline{\mathcal{H O M}}(\mathfrak{H}, \mathfrak{G})$, are isomorphic if and only if $N P$ and $N Q$ are simplicially homotopic. In other words, we have a natural bijection

$$
\pi_{0} \underline{\mathcal{H O M}}(\mathfrak{H}, \mathfrak{G}) \cong[N \mathfrak{H}, N \mathfrak{G}]_{\text {SSet }}
$$

Proof. Observe that a simplicial homotopy from $N P$ to $N Q$, that is, a simplicial map $\Delta^{1} \times N \mathfrak{H} \rightarrow \mathfrak{G}$ connecting $N P$ to $N Q$, is given by data almost identical to that required to give a weak transformation from $P$ to $Q$ (see $\S 4.2$ ), where instead of the squares of $\S 4.2$ we have to use squares of the form

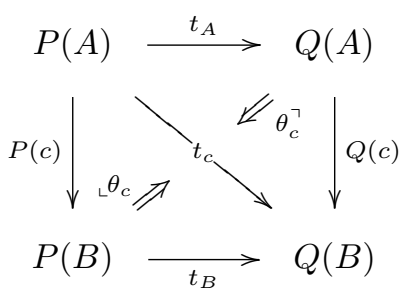

Namely, the 2-morphism $\theta_{c}$ is now replaced by a triple $\left(t_{c}, \iota_{c}, \theta_{c}^{\urcorner}\right)$. The compatibility conditions required for $\left(t_{c}, \ell_{c}, \theta_{c}^{\urcorner}\right)$are simply translated, in the obvious way, from the ones for $\theta_{c}$.

It is now easy to prove the lemma. Given a simplicial homotopy from $N P$ to $N Q$, presented by triples $\left(t_{c}, \iota_{\llcorner}, \theta_{c}^{\urcorner}\right)$, one defines $\theta_{c}:=\left[{ }_{\llcorner} \theta_{c}\right]\left[\theta_{c}^{\urcorner}\right]^{-1}$. This gives a transformation from $P$ to $Q$. Conversely, given a transformation from $P$ to $Q$, presented by $\theta_{c}$, one defines the triples $\left(t_{A} Q(c), \theta_{c}\right.$, id). This gives a simplicial homotopy from $N P$ to $N Q$. 
Proposition 7.8. Let $\mathfrak{G}$ and $\mathfrak{H}$ be 2-groupoids. Then there is a natural (up to weak transformation) equivalence of 2-groupoids

$$
\underline{\mathcal{R} \mathcal{H} o m}(\mathfrak{H}, \mathfrak{G}) \simeq \underline{\mathcal{H O M}}(\mathfrak{H}, \mathfrak{G}) .
$$

In other words, $\underline{\mathcal{H O M}}(\mathfrak{H}, \mathfrak{G})$ is a natural model for the derived hom-2-groupoid of functors from $\mathfrak{H}$ to $\mathfrak{G}$. In particular, when $\mathfrak{H}$ is cofibrant, we have a natural equivalence of 2-groupoids

$$
\underline{\mathcal{H} o m}(\mathfrak{H}, \mathfrak{G}) \simeq \underline{\mathcal{H O M}}(\mathfrak{H}, \mathfrak{G}) .
$$

Proof. First we show that if $p: \mathfrak{H}^{\prime} \rightarrow \mathfrak{H}$ is an arbitrary equivalence, then the induced map $p^{*}: \underline{\mathcal{H O M}}(\mathfrak{H}, \mathfrak{G}) \rightarrow \underline{\mathcal{H O} \mathcal{M}}\left(\mathfrak{H}^{\prime}, \mathfrak{G}\right)$ is also an equivalence. Since the induced map $N p: N \mathfrak{H}^{\prime} \rightarrow N \mathfrak{H}$ is a homotopy equivalence of Kan complexes, by Whitehead's theorem, it admits an inverse $Q: N \mathfrak{H} \rightarrow N \mathfrak{H}^{\prime}$, in the sense that $Q N p$ and $N p Q$ are homotopic to the corresponding identity maps. Since $N$ is fully faithful (Proposition 7.4), there exists a weak functor $q: \mathfrak{H} \rightarrow \mathfrak{H}^{\prime}$ such that $N q=Q$. It follows from Lemma 7.7 that $p$ and $q$ are inverse equivalences. That is, each of $f \circ q: \mathfrak{H} \rightarrow \mathfrak{H}$ and $q \circ p: \mathfrak{H}^{\prime} \rightarrow \mathfrak{H}^{\prime}$ is connected to the corresponding identity functor by a weak transformation. This implies that $p^{*}: \underline{\mathcal{H O M}}(\mathfrak{H}, \mathfrak{G}) \rightarrow \underline{\mathcal{H O M}}\left(\mathfrak{H}^{\prime}, \mathfrak{G}\right)$ and $q^{*}: \underline{\mathcal{H O M}}\left(\mathfrak{H}^{\prime}, \mathfrak{G}\right) \rightarrow \underline{\mathcal{H O M}}(\mathfrak{H}, \mathfrak{G})$ are also inverse equivalences.

To prove the proposition, we may assume now that $\mathfrak{H}$ is cofibrant. We have a natural full inclusion $\underline{\mathcal{H} o m}(\mathfrak{H}, \mathfrak{G}) \subset \underline{\mathcal{H O M}}(\mathfrak{H}, \mathfrak{G})$. So it is enough to show that the induced map $\pi_{0} \underline{\mathcal{H} o m}(\mathfrak{H}, \mathfrak{G}) \rightarrow \pi_{0} \underline{\mathcal{H O M}}(\mathfrak{H}, \mathfrak{G})$ is a bijection. Since 2Gpd is a monoidal closed model category and $\mathfrak{H}$ is cofibrant, we have that $\pi_{0} \underline{\mathcal{H} o m}(\mathfrak{H}, \mathfrak{G}) \cong$ $[\mathfrak{H}, \mathfrak{G}]_{\mathbf{2 G p d}}$. On the other hand, by Lemma 7.7 , we have that

$$
\pi_{0} \underline{\mathcal{H O M}}(\mathfrak{H}, \mathfrak{G}) \cong[N \mathfrak{H}, N \mathfrak{G}]_{\text {SSet }} .
$$

The claim now follows from the fact that $N: \mathrm{Ho}(\mathbf{2} \mathbf{G p d}) \rightarrow \mathrm{Ho}(\mathbf{S S e t})$ is an equivalence of categories (which, in particular, implies that $N$ induces a bijection between $[\mathfrak{H}, \mathfrak{G}]_{2 \text { Gpd }}$ and $\left.[N \mathfrak{H}, N \mathfrak{G}]_{\text {SSet }}\right)$.

Proposition 7.9. Given 2-groupoids $\mathfrak{G}$ and $\mathfrak{H}$, there is a natural homotopy equivalence of simplicial sets

$$
N \underline{\mathcal{R H o m}}(\mathfrak{H}, \mathfrak{G}) \simeq \operatorname{Hom}(N \mathfrak{H}, N \mathfrak{G}) .
$$

Proof. Making a cofibrant replacement for $\mathfrak{H}$ does not change either side, up to a canonical homotopy, so we may assume $\mathfrak{H}$ is cofibrant. We have to show that

$$
N \underline{\mathcal{H} o m}(\mathfrak{H}, \mathfrak{G}) \simeq \operatorname{Hom}(N \mathfrak{H}, N \mathfrak{G}) \text {. }
$$

We prove that for every simplicial set $A$, there is a natural bijection

$$
[A, N \underline{\mathcal{H} o m}(\mathfrak{H}, \mathfrak{G})]_{\text {SSet }} \cong[A, \operatorname{Hom}(N \mathfrak{H}, N \mathfrak{G})]_{\text {SSet }} .
$$

That is, the contravariant functors from SSet to Set represented by $N \underline{\mathcal{H} o m}(\mathfrak{H}, \mathfrak{G})$ and $\operatorname{Hom}(N \mathfrak{H}, N \mathfrak{G})$ are naturally isomorphic.

Since both $N \mathcal{H}$ om $(\mathfrak{H}, \mathfrak{G})$ and $\operatorname{Hom}(N \mathfrak{H}, N \mathfrak{G})$ have trivial homotopy group in degrees higher than 2 , we may assume that $A$ is a simplicial set with the same 
property. Since the unit of adjunction $A \rightarrow N W(A)$ is a weak equivalence (Theorem 5.6.iii), we may assume $A=N \mathfrak{K}$, for some cofibrant 2-groupoid $\mathfrak{K}$. We start by simplifying the right hand side:

$$
\begin{aligned}
{\left[A, N_{\mathcal{H o m}}(\mathfrak{H}, \mathfrak{G})\right]_{\mathrm{SSet}} } & =\left[N \mathfrak{K}, N_{\mathcal{H} o m}(\mathfrak{H}, \mathfrak{G})\right]_{\mathbf{S S e t}} \\
& \cong[\mathfrak{K}, \underline{\mathcal{H o m}}(\mathfrak{H}, \mathfrak{G})]_{\mathbf{2 G p d}} \\
& \cong \pi_{0} \underline{\mathcal{H o m}}(\mathfrak{K}, \underline{\mathcal{H} o m}(\mathfrak{H}, \mathfrak{G})) \\
& \cong \pi_{0} \underline{\mathcal{H o m}}(\mathfrak{K} \otimes \mathfrak{H}, \mathfrak{G}) \\
& \cong[\mathfrak{K} \otimes \mathfrak{H}, \mathfrak{G}]_{\mathbf{2 G p d}} \\
& \cong[\mathfrak{K} \times \mathfrak{H}, \mathfrak{G}]_{\mathbf{2} \mathbf{G p d}} .
\end{aligned}
$$

The next to last equality follows from the fact that in every monoidal model category, the tensor product of two cofibrant objects is cofibrant. The last equality follows from the fact that, for arbitrary 2-groupoids $\mathfrak{K}$ and $\mathfrak{H}$, the natural morphism $\mathfrak{K} \otimes \mathfrak{H} \rightarrow \mathfrak{K} \times \mathfrak{H}$ is an equivalence of 2 -groupoids (in fact it is a trivial fibration); see the last paragraph of ([La04], Section 2).

Now we do the left hand side:

$$
\begin{aligned}
{[A, \operatorname{Hom}(N \mathfrak{H}, N \mathfrak{G})]_{\mathrm{sSet}} } & =[N \mathfrak{K}, \operatorname{Hom}(N \mathfrak{H}, N \mathfrak{G})]_{\text {SSet }} \\
& \cong \pi_{0}(\operatorname{Hom}(N \mathfrak{K}, \mathbf{H o m}(N \mathfrak{H}, N \mathfrak{G})) \\
& \cong \pi_{0}(\operatorname{Hom}(N \mathfrak{K} \times N \mathfrak{H}, N \mathfrak{G})) \\
& \cong[N \mathfrak{K} \times N \mathfrak{H}, N \mathfrak{G}]_{\mathbf{S S e t}} \\
& \cong[N(\mathfrak{K} \times \mathfrak{H}), N \mathfrak{G}]_{\mathbf{S S e t}} \\
& \cong[\mathfrak{K} \times \mathfrak{H}, \mathfrak{G}]_{\mathbf{2}} \mathbf{G p d} .
\end{aligned}
$$

We see that the left hand side coincides with the right hand side. This implies that $N \underline{\mathcal{H} o m}(\mathfrak{H}, \mathfrak{G})$ and $\operatorname{Hom}(N \mathfrak{H}, N \mathfrak{G})$ are naturally isomorphic in $\mathrm{Ho}(\mathbf{S S e t})$. Since both $N \underline{\mathcal{H} o m}(\mathfrak{H}, \mathfrak{G})$ and $\operatorname{Hom}(N \mathfrak{H}, N \mathfrak{G})$ are Kan complexes, it follows from Whitehead's theorem that they are naturally homotopy equivalent.

Corollary 7.10. Let $\mathfrak{G}$ and $\mathfrak{H}$ be 2-groupoids. Then, there is a natural (up to homotopy) homotopy equivalence of simplicial sets

$$
\operatorname{Hom}(N \mathfrak{H}, N \mathfrak{G}) \simeq N \underline{\mathcal{R} \mathcal{H o m}}(\mathfrak{H}, \mathfrak{G}) \simeq N \underline{\mathcal{H O} \mathcal{M}}(\mathfrak{H}, \mathfrak{G})
$$

Proof. Combine Proposition 7.8 and Proposition 7.9.

Corollary 7.11. Let $\mathfrak{G}$ and $\mathfrak{H}$ be 2-groupoids, and let $f \in[\mathfrak{H}, \mathfrak{G}]_{\mathbf{2 G p d}}$. Then there is a weak map $\tilde{f}: \mathfrak{H} \rightarrow \mathfrak{G}$, unique up to transformation, which induces $f$ in the homotopy category.

Proof. This follows from the fact that

$$
[\mathfrak{H}, \mathfrak{G}]_{\mathbf{2} \mathbf{G p d}} \cong \pi_{0} \underline{\mathcal{R H} \text { Hom }}(\mathfrak{H}, \mathfrak{G}) \simeq \pi_{0} \underline{\mathcal{H O} \mathcal{O} \mathcal{M}}(\mathfrak{H}, \mathfrak{G}) .
$$

In ([MoSe], Proposition 2.2.i) it is claimed that the functor $W$ : SSet $\rightarrow$ 2Gpd, the left adjoint to the nerve functor, preserves products. Unfortunately this is easily 
seen to be false: $W(I \times I)$ is not isomorphic to $W(I) \times W(I)$ (but they are naturally homotopy equivalent). If this were true, an easy argument would imply that there is natural isomorphism of simplicial sets

$$
N \underline{\mathcal{H} o m}(W X, \mathfrak{G}) \cong \operatorname{Hom}(X, N \mathfrak{G}),
$$

for every simplicial set $X$ and every 2-groupoid $\mathfrak{G}$; in other words, the adjunction between $W$ and $N$ would be an enriched adjunction (which is not true either). Since $W X$ is cofibrant ([MoSe], Proposition 2.2.ii), we would indeed have

$$
N \underline{\mathcal{R H} \text { om }}(W X, \mathfrak{G}) \cong N \underline{\mathcal{H o m}}(W X, \mathfrak{G}) \cong \operatorname{Hom}(X, N \mathfrak{G}),
$$

which would lead to a straightforward proof of Proposition 7.9. ${ }^{2}$

This line of argument is unfortunately flawed. Nevertheless, one can prove the following result (which will not be needed anywhere in this paper).

Proposition 7.12. Given a 2-groupoid $\mathfrak{G}$ and a simplicial set $X$, there is a natural homotopy equivalence of simplicial sets

$$
N \underline{\mathcal{R} H o m}(W X, \mathfrak{G}) \simeq \operatorname{Hom}(X, N \mathfrak{G}) .
$$

That is, the adjunction between $N$ and $W$ can be enriched, up to homotopy.

Proof. Note that since $\mathfrak{G}$ has trivial homotopy groups in degrees higher than 2 , and since the unit of adjunction $X \rightarrow N W(X)$ induces isomorphisms on $\pi_{i}, i \leqslant 2$, the two simplicial sets $\operatorname{Hom}(X, N \mathfrak{G})$ and $\operatorname{Hom}(N W(X), N \mathfrak{G})$ are naturally homotopy equivalent. Now apply Proposition 7.9 with $\mathfrak{H}=W(X)$.

\section{3. $\quad$ Monoidal properties of $N$ and $W$}

In this subsection we say a few words about the monoidal properties of the functors $N$ and $W$. We briefly touch upon this issue without getting into much detail. The material in this subsection will not be used elsewhere in the paper.

What follows is for the most part a consequence of Tonks' Eilenberg-Zilber theory [To]. Tonks' results are, however, stated in the language of crossed complexes of groupoids (while we work with 2-groupoids). Consequently, his monoidal structure is given by the Brown-Higgins tensor product of crossed complexes (while we use the Gray tensor product of 2-groupoids). To make the passage from the category $\mathbf{C r s}$ of crossed modules in groupoids to the category $\mathbf{2 G p d}$ of 2-groupoids, we observe that the latter can be identified with a full subcategory of the former. Furthermore, there is an idempotent "truncation functor" $t: \mathbf{C r s} \rightarrow \mathbf{2} \mathbf{G p d}$ which sends the BrownHiggins tensor to the Gray tensor, namely $t\left(A \otimes_{B H} B\right)=t(A) \otimes t(B)$. Using the functor $t$ we can translate Tonks' results to the language of 2-groupoids.

Let us now outline certain monoidal features of the functors $N$ and $W$. The functors $N$ and $W$ are both lax monoidal and lax comonoidal, and these lax monidal/comonoidal structures on these functors are compatible in many ways:

\footnotetext{
${ }^{2}$ There is a similar type of error in [MoSe] in the Remark at the bottom of page 194 where the authors claim that the interval groupoid $\mathfrak{I}$ yields a cylinder object $\mathfrak{I} \times A$ for every 2 -groupoid $A$. This is false, because the map $A \coprod A \rightarrow \mathfrak{I} \times A$ is not in general a cofibration. The right thing to do would be to take $\mathfrak{I} \otimes A$. Or one could work with path objects $\mathfrak{G}^{\mathfrak{I}}:=\underline{\mathcal{H} o m}(\mathfrak{I}, \mathfrak{G})$.
} 
$W$ is lax monoidal. There are morphisms of 2-groupoids $b_{X, Y}: W(X) \otimes W(Y) \rightarrow$ $W(X \times Y)$, natural in simplicial sets $X$ and $Y$. This follows from [To], Proposition 2.6 .

$W$ is lax comonoidal. There are morphisms of 2-groupoids $a_{X, Y}: W(X \times Y) \rightarrow$ $W(X) \otimes W(Y)$, natural in simplicial sets $X$ and $Y$. This follows from [To], Proposition 2.1 .

$N$ is lax monoidal. There are morphisms of simplicial sets $a_{G, H}^{\prime}: N(G) \times N(H) \rightarrow$ $N(G \otimes H)$, natural in $G$ and $H$. This follows from [To], Corollary 2.4.

$N$ is lax comonoidal. There are morphisms of simplicial sets $b_{G, H}^{\prime}: N(G \otimes H) \rightarrow$ $N(G) \times N(H)$, natural in $G$ and $H$. To get these morphisms, simply apply $N$ to the natural morphism $G \otimes H \rightarrow G \times H$, and use the fact that $N(G \times H)=N G \times N H$.

Compatiblity. The monoidal and comonoidal structures on $W$ are related by (a straightforward modification of) the Eilenberg-Zilber theorem of Tonks ([To], Theorem 3.1): the compositions $a_{X, Y} \circ b_{X, Y}$ are equal to the identity, and the compositions $b_{X, Y} \circ a_{X, Y}$ are homotopic to identity-homotopy meaning left-homotopy, i.e., via mapping cylinders $G \rightrightarrows I \otimes G$-through homotopies $\phi_{X, Y}$ that are natural in $X$ and $Y$ and fix the image of $b$

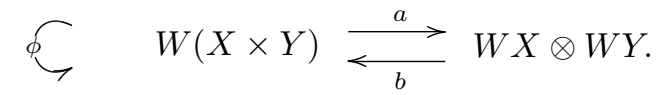

The same thing can be said about $N$. In other words, $W(X) \otimes W(Y)$ is naturally a strong deformation retract of $W(X \times Y)$, and $N(G) \times N(H)$ is naturally a strong deformation retract of $N(G \otimes H)$. In particular, all morphism $a, b, a^{\prime}$ and $b^{\prime}$ are weak equivalences.

It also seems plausible that the pair $(W, N)$ is indeed a monoidal adjunction (and also a comonoidal adjunction). One could also ask whether the monoidal (resp., comonoidal) structure of $W$ is compatible with the comonoidal (resp., monoidal) structure of $N$. One may also wonder if the above mentioned deformation retractions for $W$ are in some way compatible with the ones for $N$.

\section{Pointed weak functors and application to crossed modules}

We can prove pointed versions of the results of the previous section. For instance, we have the following propositions.

Proposition 8.1. Let $\mathfrak{G}$ and $\mathfrak{H}$ be pointed 2-groupoids. Then, there is a natural (up to homotopy) equivalence of 2-groupoids

$$
\underline{\mathcal{R H o m}}_{*}(\mathfrak{H}, \mathfrak{G}) \simeq \underline{\mathcal{H O}}_{*}(\mathfrak{H}, \mathfrak{G}) .
$$

In other words, $\underline{\mathcal{H O M}}_{*}(\mathfrak{H}, \mathfrak{G})$ is a natural model for the derived groupoid of pointed functors from $\mathfrak{H}$ to $\mathfrak{G}$. In particular, when $\mathfrak{H}$ is cofibrant, we have a natural equivalence of 2-groupoids

$$
\underline{\mathcal{H o m}}_{*}(\mathfrak{H}, \mathfrak{G}) \simeq \underline{\mathcal{H O M}}_{*}(\mathfrak{H}, \mathfrak{G}) .
$$


Proposition 8.2. Let $\mathfrak{G}$ and $\mathfrak{H}$ be pointed 2-groupoids. Then, there is a natural (up to homotopy) homotopy equivalence of simplicial sets

$$
\operatorname{Hom}_{*}(N \mathfrak{H}, N \mathfrak{G}) \simeq N \underline{\mathcal{R H o m}}_{*}(\mathfrak{H}, \mathfrak{G}) \simeq N \underline{\mathcal{H O}}_{*}(\mathfrak{H}, \mathfrak{G}) .
$$

Proof. We prove that whenever $\mathfrak{H}$ is a cofibrant pointed 2-groupoid and $\mathfrak{G}$ an arbitrary pointed 2-groupoid, then we have a natural homotopy equivalence

$$
N \underline{\mathcal{H o m}}_{*}(\mathfrak{H}, \mathfrak{G}) \simeq \operatorname{Hom}_{*}(N \mathfrak{H}, N \mathfrak{G}) .
$$

We will always denote the base points by $*$. Consider the map of 2 -groupoids

$$
\underline{\mathcal{H o m}}(\mathfrak{H}, \mathfrak{G}) \stackrel{\mathrm{ev}_{*}}{\longrightarrow} \mathfrak{G} .
$$

Applying Axiom M7 of monoidal closed model categories ([La04], 7) to the cofibration $* \rightarrow \mathfrak{H}$ and fibration $\mathfrak{G} \rightarrow *$, we see that $\mathrm{ev}_{*}$ is a fibration of 2 -groupoids. Similarly, using the fact that the nerve of a 2-groupoid is fibrant (Proposition 5.4.ii), together with SM7 for SSet, we see that the following map of simplicial sets is also a fibration:

$$
\operatorname{Hom}(N \mathfrak{H}, N \mathfrak{G}) \stackrel{\mathrm{ev}_{*}}{\longrightarrow} N \mathfrak{G} .
$$

Now, we observe that $\underline{\mathcal{H o m}}_{*}(\mathfrak{H}, \mathfrak{G})$ is the fiber over $*$ of ev $_{*}: \underline{\mathcal{H} o m}(\mathfrak{H}, \mathfrak{G}) \rightarrow \mathfrak{G}$, and $\operatorname{Hom}_{*}(N \mathfrak{H}, N \mathfrak{G})$ is the fiber over $*$ of $\mathrm{ev}_{*}: \operatorname{Hom}(N \mathfrak{H}, N \mathfrak{G}) \rightarrow N \mathfrak{G}$. The claim now follows from Proposition 7.9, plus the fact that taking nerves preserves weak equivalences and fiber products (Proposition 5.4.i,ii).

Proposition 8.3. Let $\mathfrak{G}$ and $\mathfrak{H}$ be pointed 2-groupoids. Then, for every $f \in$ $[\mathfrak{H}, \mathfrak{G}]_{\mathbf{2}} \mathbf{G p d}_{*}$ there is a weak pointed map $\tilde{f}: \mathfrak{H} \rightarrow \mathfrak{G}$, unique up to pointed transformation, which induces $f$ in the homotopy category.

Using the fact that $\mathbf{2} \mathbf{G p}$ is a full subcategory of $\mathbf{2} \mathbf{G} \mathbf{p d} \mathbf{d}_{*}$, we immediately obtain the 2-group versions of the above propositions. In particular, using the fact that CrossedMod and 2Gp are equivalent (§3.3), we get the corresponding statements for crossed modules as well. Note that in this case both sides of the equivalence in Proposition 8.1 are actually 1-groupoids.

For the readers' information we include the crossed module translations of the notions of weak functor between 2-group, transformation between weak functors, and modification between transformations.

Definition 8.4. Let $\mathfrak{G}$ and $\mathfrak{H}$ be crossed modules. A weak map $P: \mathfrak{H} \rightarrow \mathfrak{G}$ consists of the following data:

- a group homomorphism $p_{2}: H_{2} \rightarrow G_{2}$,

- a set map $p_{1}: H_{1} \rightarrow G_{1}$,

- a set map $\varepsilon: H_{1} \times H_{1} \rightarrow G_{2}$, denoted by $(x, y) \mapsto \varepsilon_{x, y}$.

These data should satisfy the following conditions:

W1. $p_{1}(1)=1, \varepsilon_{1,1}=1$;

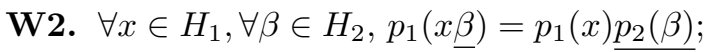

W3. $\forall x, y \in H_{1}, p_{1}(x) p_{1}(y) \underline{\varepsilon_{x, y}}=p_{1}(x y)$; 
W4. $\forall x, y, z \in H_{1}, \varepsilon_{x, y}^{p_{1}(z)} \varepsilon_{x y, z}=\varepsilon_{y, z} \varepsilon_{x, y z}$;

W5. Equivariance:

$$
\forall x \in H_{1}, \forall \beta \in H_{2}, \quad \varepsilon_{x^{-1}, x} p_{2}\left(\beta^{x}\right)=p_{2}(\beta)^{p_{1}(x)} \varepsilon_{\underline{\beta}, x} \varepsilon_{x^{-1}, \underline{\beta} x} .
$$

Definition 8.5. Suppose we are given two weak functors $P=\left(p_{1}, p_{2}, \epsilon\right)$ and $Q=$ $\left(q_{1}, q_{2}, \delta\right)$ from $\mathfrak{H}$ to $\mathfrak{G}$, as in Definition 8.4. A transformation from $P$ to $Q$ is given by a pair $(a, \theta)$, where $a \in G_{1}$, and $\theta: H_{1} \rightarrow G_{2}$ is a map of sets. This pair should satisfy the following conditions:

T0. $\varepsilon_{x, y}^{a} \theta(x y)=\theta(x)^{p_{1}(y)^{a}} \theta(y) \delta_{x, y}$, for every $x, y \in H_{1}$;

T1. $p_{1}(x)^{a} \theta(x)=q_{1}(x)$, for every $x \in H_{1}$;

T2. $p_{2}(\alpha)^{a} \theta(\underline{\alpha})=q_{2}(\alpha)$, for every $\alpha \in H_{2}$.

Such a transformation is called pointed if $a=1$.

Definition 8.6. A modification between two transformations $(a, \theta)$ and $(b, \sigma)$ is given by an element $\mu \in G_{2}$ that has the following properties:

M1. $a \mu=b$;

M2. $\mu \sigma(x)=\theta(x) \mu^{q_{1}(x)}$, for every $x \in H_{1}$.

By definition, the only pointed modification is $\mu=1$, namely the trivial modification.

Remark 8.7. The axioms given in the above three definitions are obtained through tedious calculations - in which various terms magically cancel at the end - and we do not claim that their equivalence to the axioms for a 2-groupoid is obvious by any means.

Crossed modules are in practice more amenable to computations than 2-groups, whereas 2-groups are more conceptual. An important problem regarding 2-groups is to compute the derived hom-2-groupoids $\underline{\mathcal{H} H o m}(\mathfrak{H}, \mathfrak{G})$ in an explicit way. Of course, one could use obstruction theory to provide a description of elements of $\underline{\mathcal{R H} \text { Hom }}(\mathfrak{H}, \mathfrak{G})$ using cohomological invariants, but this may in practice be not so useful. In [No] such an explicit description is given using the notion of a papillon between two crossed modules (see loc. cit. Definition 11.1 and Theorem 11.3). The main tool is to translate the problem to the language of crossed modules, using the three definitions give above, and then work things out by hand using some simple group theoretic constructions.

\section{Weak 2-groupoids}

In Section 4 we saw (Proposition 5.4) that the nerve functor $N: \mathbf{2 G p d} \rightarrow \mathbf{S S e t}$ identifies $\mathbf{2 G p d}$ with a subcategory of SSet that is not full. The lack of fullness was remedied in Section 7 by considering weak functors between 2-groupoids (Proposition 7.4). So the category $\mathbf{2} \mathbf{G p d}_{w}$ is identified with a certain full subcategory of SSet. The natural question now is to characterize this full subcategory. We know 
so far that a simplicial set $X$ in the image of $N$ has the following properties:

1. $X$ is a Kan complex.

2. $X$ is 3 -coskeletal (i.e., $X$ is isomorphic to the coskeleton of its 3 -truncation $\left\{X_{0}, X_{1}, X_{2}, X_{3}\right\}$.

Is this enough to characterize the image of $N$ ? The answer is no. To see what is missing, let us consider the nerve functor from Gpd to SSet. In this case, every simplicial set in the image of $N$ is Kan and 2-coskeletal. But a simplicial set in the image of $N$ has an additional property: it satisfies the condition for a minimal simplicial set for $n \geqslant 1$. That is, such $X$ is minimal, except possibly at degree 0 .

Definition 9.1. We say a simplicial set $X$ is $k$-minimal if it satisfies the minimality condition for $n \geqslant k$. That is, every two $n$-simplices that have equal boundaries and are homotopic relative to their common boundary are actually equal (see[Ma], $\S 9$, or [GoJa], §I.10).

For example, the nerve of a groupoid is 1-minimal, as we explained above.

It is easy to see that any $X$ that is isomorphic to the nerve of a 2 -groupoid satisfies the following property:

3. $X$ is 2-minimal.

\section{Remark 9.2.}

a. Any $k$-coskeletal $X$ is automatically $(k+1)$-minimal. So, any 3 -skeletal $X$ is 4-minimal. Therefore, the condition (3) puts only two new restrictions on $X$. In other words, in the presence of $(\mathbf{1})$ and $(\mathbf{2})$, we only need to verify that the condition of Definition 9.1 is satisfied with $n=2$ and $n=3$.

b. If $X$ is Kan and 2-minimal, then it can be shown that for any $n \geqslant 3$, whenever two $n$-simplices in $X$ have the same boundary, then they are indeed homotopic relative boundary (hence equal). In other words, the natural map $X \rightarrow \operatorname{Cosk}_{2}(X)$ is injective (in general, $k$-minimal implies $X \rightarrow \operatorname{Cosk}_{k} X$ injective). So, one of the conditions in (a) can be replaced by the injectivity of $X \rightarrow \mathrm{Cosk}_{2} X$.

Let SSet2 denote the full-subcategory of SSet consisting of simplicial sets satisfying conditions (1), (2) and (3) above. It is more natural now to consider the (fully faithful) functor $N: \mathbf{2} \mathbf{G p d}_{w} \rightarrow \mathbf{S S e t} \mathbf{2}$ and ask whether it is an equivalence. The answer is no! In the same way we had to weaken the notion of a functor in order to make $N$ full, we have to weaken the notion of a 2-groupoid to make $N$ essentially surjective.

Definition 9.3. A weak 2-category $\mathfrak{C}$ is defined by exactly the same data and axioms as a strict 2-category, except that the axiom on the associativity of composition of three composable arrows $a, b$ and $c$ is replaced by the choice of an associator $\phi_{a, b, c}:(a b) c \Rightarrow a(b c)$, for every such triples of arrows. ${ }^{3}$ The associators are required

\footnotetext{
${ }^{3}$ The properties of the identity arrows, however, remain strict. In this sense, our notion of weak is stronger than the usual one.
} 
to satisfy the following conditions:

A1. The famous pentagon identity

$$
\left[\phi_{a, b, c} d\right]\left[\phi_{a, b c, d}\right]\left[a \phi_{b, c, d}\right]=\left[\phi_{a b, c, d}\right]\left[\phi_{a, b, c d}\right] .
$$

See, for example ([Be], page 6).

A2. Whenever one of $a, b$ or $c$ is the identity, then $\phi_{a, b, c}$ is the identity.

A weak 2-groupoid is a weak 2-category in which for every arrow $a$ there exists an arrow $b$, with reverse source and target as $a$, such that $a b$ and $b a$ are 2-isomorphic to the corresponding identity arrows. A weak 2-group is a weak 2-groupoid with one object.

Remark 9.4. The requirement on inverses seems a little too weak, as we have not asked for any compatibility with the associators. However, as is shown in [BaLa] for the case of weak 2-groups, it can be shown that one can select a coherent family of inverses in a way that all the required compatibility conditions with the associators are satisfied. We will not, however, need this fact here.

\subsection{Weak functors between weak 2-categories, and transformations}

We can talk about weak functors between weak 2-categories. The definition is the same as Definition 7.1, but the square should be replaced by the following hexagon

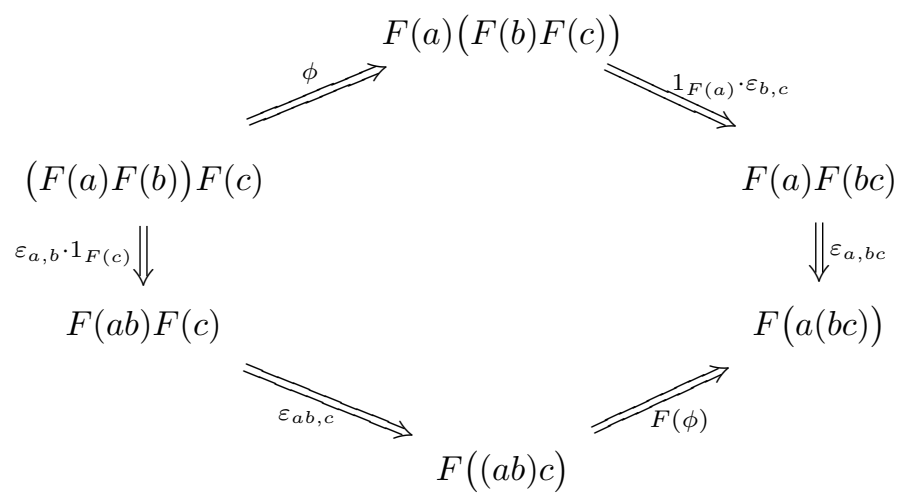

Weak 2-categories and weak 2-functors between them form a category, which we denote by W2Cat.

We can also talk about transformations between weak functors, and modifications between them. The definitions are exactly the same as the ones for the case of weak functors between strict 2-categories ( $\$ 7.1)$.

Similarly, we have the categories W2Gpd and W2Gp. (We define weak 2functors between weak 2-group(oid)s to be simply weak 2 -functors between the underlying weak 2-categories.)

\subsection{Nerve of a weak 2-groupoid}

To a weak 2-category $\mathfrak{C}$ we can associate a nerve $N \mathfrak{C}$. The simplices are exactly the same as in the case of strict 2-categories (see Section 4), except that now the 
commutativity of a 3-cell like the one on page 83 means $\left[\phi_{f, g, m}\right][f \gamma][\beta]=[\alpha m][\delta]$. With some careful, and not entirely easy, 2-diagram chasing, it can be shown that taking nerves is a functor $N: \mathbf{W} \mathbf{G} \mathbf{G p d} \rightarrow \mathbf{S S e t 2}$. This functor is indeed an equivalence of categories (Proposition 9.6).

Some notation. In what follows, the symbols $d_{i}, s_{j}$ stand for the face and degeneracy maps of a simplicial set. The boundary of $\Delta^{n}$ is denoted by $\partial \Delta^{n}$. The $k^{\text {th }}$ horn $\Lambda_{k}^{n}$ of $\Delta^{n}$ is the subcomplex of the simplicial set $\Delta^{n}$ generated by all but the $k^{t h}(n-1)$ faces. If $x \in X_{n}$ is an n-simplex of a simplicial set $X$, we use the same notation for the corresponding map $x: \Delta^{n} \rightarrow X$.

Lemma 9.5. Let $X$ be a simplicial set in $\mathbf{S S e t 2 . ~ L e t ~} x, y: \Delta^{3} \rightarrow X$ be 3-simplices that are equal on a horn $\Lambda_{k}^{3}$, for some $0 \leqslant k \leqslant 3$. Then $x=y$.

Proof. It is easy to see that $d_{k}(x)$ and $d_{k}(y)$ are equal on the boundary, and are homotopic relative to their equal boundary. So, by 2-minimality, $d_{k}(x)=d_{k}(y)$. It follows from Remark 9.2.b that $x=y$.

Proposition 9.6. The functor $N: \mathbf{W} 2 \mathbf{G p d} \rightarrow \mathbf{S S e t 2}$ is an equivalence of categories.

Sketch. We explain how to construct an inverse equivalence SSet2 $\rightarrow$ W2Gpd. Pick a simplicial set $X \in \mathbf{S S e t 2}$. Define the weak 2-groupoid $\mathfrak{G}_{X}$ as follows. The set objects of $\mathfrak{G}_{X}$ is $X_{0}$, and the set of arrows is $X_{1}$, with the source and target maps being $s=d_{0}, t=d_{1}$. Take the degenerate 1-simplices in $X_{1}$ for the identity arrows. To define composition of arrows, take two composable arrows $f, g \in \mathfrak{G}_{X}$, and consider the induced map $\Lambda_{2}^{1} \rightarrow X$. Since $X$ is Kan, we can extend this map to a map $I_{f, g}: \Delta^{2} \rightarrow X$; choose such an extension. Make sure to choose $I_{f, g}=s_{0}(f)$, respectively $I_{f, g}=s_{1}(g)$, when $g$, respectively $f$, is an identity arrow (that is, a degenerate 1-simplex in $X_{1}$ ). Define the composition $f g$ to be the restriction of $I_{f, g}$ to the $1^{\text {st }}$ face of $\Delta^{2}$. That is, $f g:=d_{1}\left(I_{f, g}\right)$.

For a pair of arrows $f$ and $g$ between objects $A$ and $B$, we define the set of 2-morphism from $f$ to $g$ to be the set of 2-simplices $\alpha \in X_{2}$ such that $d_{2}(\alpha)=f$, $d_{1}(\alpha)=g$, and $d_{0}(\alpha)=\operatorname{id}_{B}$. When $f=g$, we take the degenerate 2-simplex $s_{1}(f)$ for the identity 2-morphism from $f$ to itself .

Using the fact that $X$ is Kan, plus the fact that $X$ satisfies the minimality assumption (for 2-simplices), one can show, for given objects $A$ and $B$, that there is a well-defined associative composition for 2-morphisms, making $\operatorname{Hom}(A, b)$ into a groupoid. The identity arrows in this hom-groupoid are the identity 2-morphisms defined in the previous paragraph. One can also prove the following key fact:

- Given 1-morphisms $f, g$ and $h$, the 2-morphisms from $f g$ to $h$ are in a natural bijection with the 2-simplices $\alpha \in X_{2}$ such that $f=d_{2}(\alpha), g=d_{0}(\alpha)$ and $h=$ $d_{1}(\alpha)$; when $h=f g$, the 2-isomorphism id: $f g \rightarrow h$ corresponds to $I_{f, g}$ under this bijection.

Let us explain how to compose a 2-morphism with a 1-morphism. For example, suppose $f$ and $g$ are 1-morphisms from $A$ to $B, \alpha: f \Rightarrow g$ is a 2-morphism (as defined above), and $h$ is a 1-morphism from $B$ to another object $C$. We define 
$\alpha h: f h \Rightarrow g h$ as follows. Let $H: \Lambda_{2}^{3} \rightarrow X$ be the horn defined by $d_{0}(H)=s_{0}(h)$, $d_{1}(H)=I_{g, h}, d_{3}(H)=\alpha$. By the Kan property of $X$, together with Lemma 9.5, we obtain a unique extension of $H$ to a 3 -simplex $\bar{H}: \Delta^{3} \rightarrow X$. The second face $d_{2}(\bar{H})$ of this 3 -simplex determines, by - a 2 -morphism $f h \Rightarrow g h$, which we take to be $\alpha h$.

Given three composable 1-morphisms $f, g$, and $h$, we define the associator $\phi_{f, g, h}$ as follows. Consider the horn $H: \Lambda_{1}^{3} \rightarrow X$ defined by $d_{0}(H)=I_{g, h}, d_{2}(H)=I_{f, g h}$, and $d_{3}(H)=I_{f, g}$. Let $\bar{H}$ be the (unique) extension of $H$ to a 3-simplex $\bar{H}: \Delta^{3} \rightarrow X$. We define $\phi_{f, g, h}:(f g) h \Rightarrow f(g h)$ to be the 2 -simplex $d_{1}(\bar{H})$; here, we are again using $\longrightarrow$. The following fact is the key to proving that $\mathfrak{G}_{X}$ satisfies the axioms of a weak 2-groupoid, as well as to proving that the functor $X \mapsto \mathfrak{G}_{X}$ is an inverse to the nerve functor.

- Suppose we are given a hollow 3-simplex $\partial \Delta^{3} \rightarrow X$ whose faces are labeled as in the tetrahedron on page 83 . Then, this hollow 3 -simplex can be extended to a (necessarily unique) 3 -simplex $\Delta^{3} \rightarrow X$ if an only if $(f \gamma)(\beta)=(\alpha m)(\delta)$.

Using $\longrightarrow$ we prove the pentagon identity as follows. Let $\{a, b, c, d\}$ be a sequence of composable maps. We will denote the sequence of vertices of these arrows by $\{0,1,2,3,4\}$. Let $\Lambda \subset \Delta^{4}$ be the 2 -skeleton of the standard 4 -simplex. Consider the map $J: \Lambda \rightarrow X$ whose effect on 2 -faces is given by:

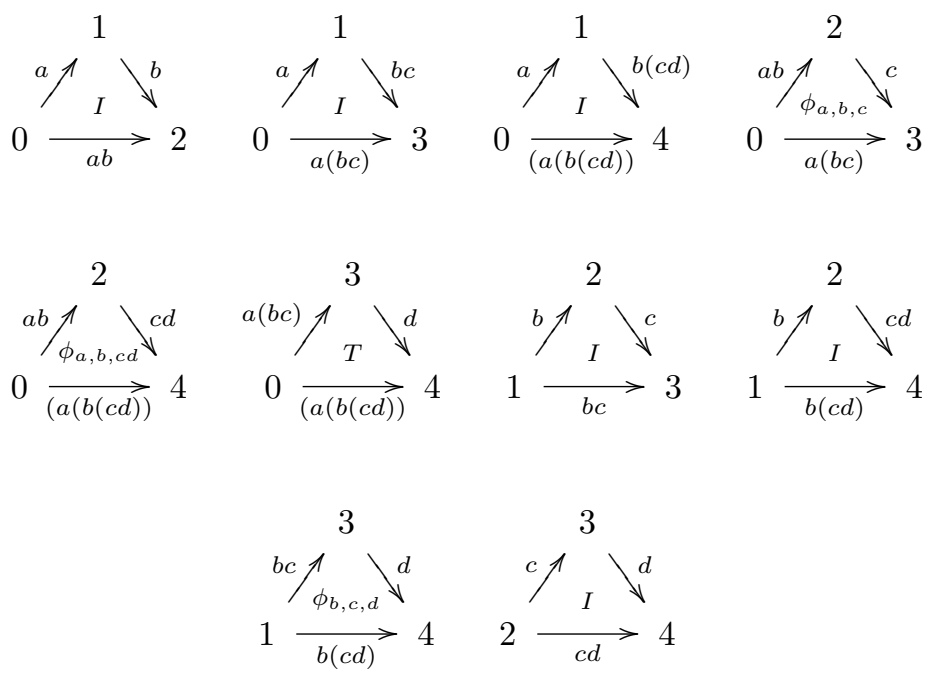

In the above diagrams, $I_{f, g}$ has been abbreviated to $I$ (which is meant to remind the reader of the identity 2-morphism), and $T=\left(\phi_{a, b c, d}\right)\left(a \phi_{b, c, d}\right)$.

In fact, $J$ extends (uniquely) to the horn $H: \Lambda_{1}^{5} \rightarrow X$. To see this, one just needs to verify that $J$ can be extended to each of the faces $d_{0}, d_{2}, d_{3}$, and $d_{4}$, which is easily done using $\rightarrow$. The horn $H$ can now be uniquely extended to a full 4 -simplex $\bar{H}: \Delta^{4} \rightarrow X$ using the Kan extension property and Remark 9.2.b. This in particular implies that the, a priori hollow, 3-cell $d_{1}(\bar{H})$ has a filling. Using $\bullet$, this translates 
to

$$
\left(\phi_{a, b, c} d\right)\left(\phi_{a, b c, d}\right)\left(a \phi_{b, c, d}\right)=\left(\phi_{a b, c, d}\right)\left(\phi_{a, b, c d}\right)
$$

which is the desired pentagon identity. This completes the (sketch of the) proof that $\mathfrak{G}_{X}$ is a weak 2-groupoid.

Let us now verify the functoriality of our construction. Namely, given a simplicial map $F: X \rightarrow Y$ in SSet2, we describe the induced weak functor $\mathfrak{G}_{F}: \mathfrak{G}_{X} \rightarrow \mathfrak{G}_{Y}$. The effect of $\mathfrak{G}_{F}$ on objects, 1-morphisms and 2-morphisms is defined in the obvious way. Given a pair $f$ and $g$ of composable morphisms in $\mathfrak{G}_{X}$, we define $\epsilon_{f, g}$ to be the 2-morphism in $\mathfrak{G}_{Y}$ associated to the 2-simplex $F\left(I_{f, g}\right)$; we are using $\square$ again.

The fact that the above two functors are inverse to each other is not difficult to check using and

Definition 5.2 and Lemma 7.6 remain valid for weak maps between weak 2groupoids.

\section{3. $\quad$ Strictifying weak 2-groupoids}

Most of the 2-groups that appear in nature are weak. There are, however, general methods to strictify weak 2-groups. We briefly address this problem after the following example. We point out that the question of strictifying weak 2-groups, or more generally bicategories, has been studied by many authors (see for instance, $[\mathbf{M c P a}],[\mathbf{L a p}],[\mathbf{B a L a}],[\mathbf{L a 0 4}])$, but our point of view is slightly different, in that our main emphasis is on showing that the strictifications that we consider do respect derived mapping spaces. In other words, our main interest is in using strictifications (or weakenings, for that matter) as tools for computing derived mapping spaces.

Example 9.7. Let $A$ be an abelian group, and $\Gamma$ an arbitrary group. Consider the groupoid $\mathfrak{E}$ of central extensions of $\Gamma$ by $A$. There is a natural multiplication on $\mathfrak{E}$ which makes it into a weak 2-group. It is defined as follows. Take two objects

$$
\begin{aligned}
& \epsilon_{1}: 1 \longrightarrow A \stackrel{\alpha}{\longrightarrow} E_{1} \longrightarrow \Gamma \longrightarrow 1 \\
& \epsilon_{2}: 1 \longrightarrow A \stackrel{\beta}{\longrightarrow} E_{2} \longrightarrow \Gamma \longrightarrow 1
\end{aligned}
$$

in $\mathcal{E}(\Gamma, A)$. The sum $\epsilon_{1}+\epsilon_{2}$ is the sequence

$$
1 \longrightarrow A \stackrel{\left(\alpha, \beta^{-1}\right)}{\longrightarrow} E_{1} \underset{\Gamma}{\stackrel{A}{\times}} E_{2} \longrightarrow \Gamma \longrightarrow 1,
$$

where $\beta^{-1}$ stands for the point-wise inversion of $\beta$. The inverse of $\epsilon_{1}$ is given by

$$
\epsilon_{1}^{-1}: 1 \longrightarrow A \stackrel{\alpha^{-1}}{\longrightarrow} E_{1} \longrightarrow \Gamma \longrightarrow 1 \text {. }
$$

The set of isomorphism classes of elements in $\mathcal{E}(\Gamma, A)$ is in natural bijection with $H^{2}(\Gamma, A)$, and the automorphism group of an object in $\mathfrak{E}$ is naturally isomorphic to $H^{1}(\Gamma, A)$. Therefore, we have $\pi_{1} \mathfrak{E}=H^{2}(\Gamma, A)$, and $\pi_{2} \mathfrak{E}=H^{1}(\Gamma, A)$.

We can give a strict model for the weak 2-group $\mathfrak{E}$ using cocycles as follows. Let $C^{2}(\Gamma, A)$ be the group of 2-cocycles from $\Gamma$ to $A$. Let $A^{\Gamma}$ be the group of (set) maps from $\Gamma$ to $A$. Let $C^{2}(\Gamma, A)$ act trivially on $A^{\Gamma}$. Form the crossed module $\left[\partial: A^{\Gamma} \rightarrow C^{2}(\Gamma, A)\right]$, where $\partial$ is the boundary map. This crossed module is a strict 
model for $\mathfrak{E}$. In fact, this example can be made to work in the case where $A$ is an arbitrary $\Gamma$-module, and $\mathfrak{E}$ is the group of such extensions for which the induced action of $\Gamma$ on $A$ coincides with the given one.

Now we consider the general strictification problem for weak 2-groupoids and weak maps. We have a hierarchy of 2 -groupoids as in the following commutative diagram:

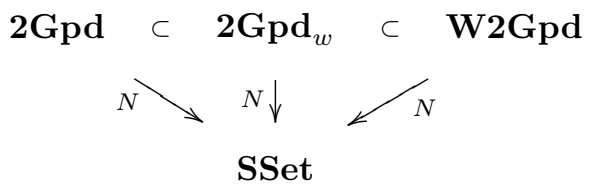

All the maps in this diagram respect (and reflect) weak equivalences. So we can pass to homotopy categories.

Proposition 9.8. We have a commutative diagram of equivalences of categories

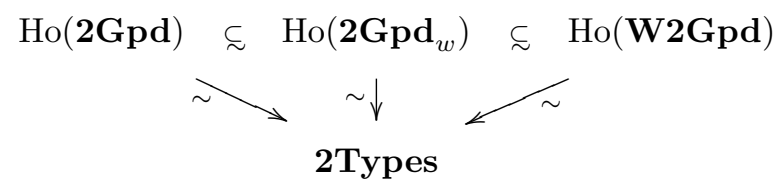

Here 2Types stands for the full subcategory of $\mathrm{Ho}(\mathbf{S S e t})$ consisting of all simplicial sets $X$ with $\pi_{i} X=0, i \geqslant 3$. In all three cases, the downward arrows are given by $N$, and their inverse equivalences are given by $W$.

Proof. We have to prove that $W$ provides an inverse equivalence to all of the downward arrows. For the leftmost arrow, this is part of Theorem 5.6. Let us prove the statement for the rightmost arrow. We have to show that $W: \mathbf{2 T y p e s} \rightarrow$ $\mathrm{Ho}(\mathbf{W} \mathbf{2 G} \mathbf{p d})$ is an inverse equivalence to $N: \mathrm{Ho}(\mathbf{W} \mathbf{2 G} \mathbf{p d}) \rightarrow$ 2Types. The fact that $N \circ W$ : 2Types $\rightarrow \mathbf{2 T y p e s}$ is equivalent to $\mathrm{id}_{\mathbf{2} \text { Types }}$ is already part of Theorem 5.6, because $W$ : 2Types $\rightarrow \mathrm{Ho}(\mathbf{W} \mathbf{2 G p d})$ factors through $\mathrm{Ho}(\mathbf{2 G} \mathbf{p d})$. We now show that for every weak 2-groupoid $\mathfrak{G}$ there is a weak equivalence $\mathfrak{G} \rightarrow W N(\mathfrak{G})$, natural in $\mathfrak{G}$. Since $N: \mathbf{W} \mathbf{2 G p d} \rightarrow$ SSet is fully faithful and reflects weak equivalences, it is enough to construct a weak equivalence $N \mathfrak{G} \rightarrow N W N(\mathfrak{G})$. But this is easy because we know that for every simplicial set $X$ with $\pi_{i} X=0$ for $i \geqslant 3$, for example $X=N \mathfrak{G}$, there is a natural map $X \rightarrow N W(X)$, namely the unit of the adjunction of Theorem 5.6, and that this map is a weak equivalence (Theorem 5.6.iii).

The proof for the case of $\mathrm{Ho}\left(\mathbf{2} \mathbf{G} \mathbf{p d} \mathbf{d}_{w}\right)$ is similar.

The same discussion applies to the case where everything is pointed. In particular, 
we have a diagram

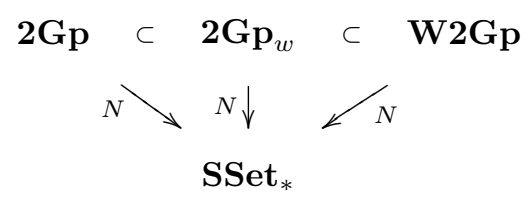

which passes to the homotopy category, and we obtain the following proposition whose proof is similar to that of Proposition 9.8.

Proposition 9.9. We have a commutative diagram of equivalences of categories

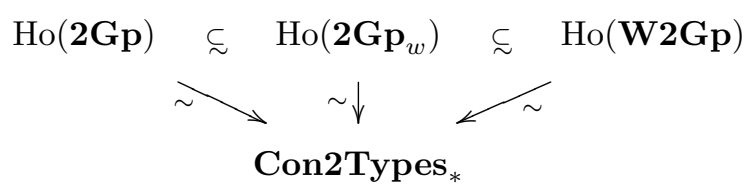

Here Con2 Types $_{*}$ is the full subcategory of $\mathrm{Ho}\left(\mathbf{S S e t}_{*}\right)$ consisting of pointed connected simplicial sets $X$ with $\pi_{i} X=0, i \geqslant 3$. In all three cases, the downward arrows are given by $N$, and their inverse equivalences are given by $W$.

Propositions 9.8 and 9.9 can in fact be strengthened to statements about simplicial mapping spaces. This means that all these functors respect derived mapping spaces, up to natural homotopy equivalences. Let us explain what we mean by "derived." If we are in $\mathbf{2} \mathbf{G p d}, \mathbf{2} \mathbf{G p d}_{*}$ or $\mathbf{2} \mathbf{G p}$, derived simply means working with $\underline{\mathcal{R H}}$ om rather than $\mathcal{H o m}$. If we are in SSet, to compute derived mapping spaces we need to first make a fibrant replacement on the target; observe, however, that the images of the nerve functors always land in the fibrant part, so in such cases derived mapping spaces are the same as the usual mapping spaces Hom. In $\mathbf{2} \mathbf{G p d}{ }_{w}$, W2Gpd, and in their pointed versions, derived mapping space simply means the hom-2-groupoid $\mathcal{H O M}$.

For instance, if we are given weak 2-groups $\mathfrak{H}$ and $\mathfrak{G}$, then the hom-2-groupoid $\underline{\mathcal{H O M}}(\mathfrak{H}, \mathfrak{G})$ is naturally equivalent to the derived hom-2-groupoid $\underline{\mathcal{R H} \text { Hom }}\left(\mathfrak{H}^{s}, \mathfrak{G}^{s}\right)$, where $\mathfrak{G}^{s}:=W N \mathfrak{G}$ is the "strictification" of $\mathfrak{G}$. This hom-2-groupoid is in turn naturally equivalent to $\underline{\mathcal{H O} \mathcal{M}}\left(\mathfrak{H}^{s}, \mathfrak{G}^{s}\right)$, where the latter is computed in $\mathbf{2} \mathbf{G} \mathbf{p d} \mathbf{d}_{w}$.

Proposition 9.10. Suppose $\mathfrak{G}$ and $\mathfrak{H}$ are weak 2-groupoids.

i. Let $\mathfrak{H}^{\prime} \rightarrow \mathfrak{H}$ and $\mathfrak{G} \rightarrow \mathfrak{G}^{\prime}$ be equivalences of weak 2-groupoids. Then the induced functor $\underline{\mathcal{H O M}}(\mathfrak{H}, \mathfrak{G}) \rightarrow \underline{\mathcal{H O M}}\left(\mathfrak{H}^{\prime}, \mathfrak{G}^{\prime}\right)$ is an equivalence of weak 2-groupoids.

ii. We have a natural equivalence of weak 2-groupoids

$$
\underline{\mathcal{R H o m}}\left(\mathfrak{H}^{\prime}, \mathfrak{G}^{\prime}\right) \simeq \underline{\mathcal{H O M}}(\mathfrak{H}, \mathfrak{G}),
$$

where $\mathfrak{G} \sim \mathfrak{G}^{\prime}$ and $\mathfrak{H}^{\prime} \stackrel{\sim}{\mathfrak{H}}$ are arbitrary strictifications of $\mathfrak{H}$ and $\mathfrak{G}$; no requirement on these maps being cofibration or fibration. 
iii. We have natural equivalences of simplicial sets

$$
\operatorname{Hom}(N \mathfrak{H}, N \mathfrak{G}) \simeq N \underline{\mathcal{R H} o m}\left(\mathfrak{H}^{\prime}, \mathfrak{G}^{\prime}\right) \simeq N \underline{\mathcal{H O} \mathcal{M}}(\mathfrak{H}, \mathfrak{G}),
$$

where $\mathfrak{G}^{\prime}$ and $\mathfrak{H}^{\prime}$ are as in part (ii).

Proof. Part (i) is proved by an argument similar to the one used in the proof of Proposition 7.8. Part (ii) follows from part (i) and Proposition 7.8. The second equivalence of (iii) follows from (ii). For the first equivalence, note that, since the nerve of a 2 -groupoid is a fibrant simplicial set, we have $\operatorname{Hom}(N \mathfrak{H}, N \mathfrak{G}) \simeq$ $\operatorname{Hom}\left(N \mathfrak{H}^{\prime}, N \mathfrak{G}^{\prime}\right)$. Now use Corollary 7.10.

Corollary 9.11. Suppose $\mathfrak{G}$ and $\mathfrak{H}$ are weak 2-groupoids. Then, for every map $f \in$ $[\mathfrak{H}, \mathfrak{G}]_{\text {W2Gpd, }}$, there is a weak map $\tilde{f}: \mathfrak{H} \rightarrow \mathfrak{G}$, unique up to transformation, which induces $f$ in the homotopy category.

Proof. Using Proposition 9.10, the proof of Corollary 7.11 works in this case as well.

Proposition 9.12. The pointed versions of Proposition 9.10 and Corollary 9.11 are true. In particular, we have similar statements for 2-groups and crossed modules.

\section{References}

[BaLa] J. Baez and A. Lauda, Higher dimensional algebra V: 2-groups, Theory Appl. Categ. 12 (2004), 423-491.

[Ban] Webpage of the Bangor Research Group: http://www.informatics.bangor.ac.uk/public/mathematics/ research/cathom/cathom2.html

[Ba] H-J. Baues, Combinatorial homotopy and 4-dimensional complexes, de Gruyter Expositions in Mathematics 2, Walter de Gruyter, Berlin, 1991.

[Be] J. Bénabou, Introduction to bicategories, Reports of the Midwest Category Seminar, Lecture Notes in Mathematics, vol. 47, 1-77, SpringerVerlag, New York, 1967.

[Br] K. Brown, Cohomology of Groups, Graduate Texts in Mathematics 87, Springer-Verlag, New York, 1994.

[BrGo] R. Brown, M. Golasinski, A model structure for the homotopy theory of crossed complexes, Cahiers Topologie Géom. Différentielle Catég. 30 (1989), no. 1, 61-82.

[Gr] J.W. Gray, Formal category theory: adjointness for 2-categories, Lecture Notes in Mathematics, vol. 391, Springer-Verlag, Berlin-New York, 1974.

[GoJa] P. G. Goerss and J. F. Jardine, Simplicial homotopy theory, Progress in Mathematics 174, Birkhäuser Verlag, Basel, 1999.

[La02] S. Lack, A Quillen model structure for 2-categories, K-Theory 26 (2002), 171-205.

[La04] S. Lack, A Quillen model structure for bicategories, K-Theory 33 (2004), 185-197. 
[Lap] M. L. Laplaza, Coherence for categories with group structure: an alternative approach, J. Algebra 84 (1983), no. 2, 305-323.

[Lo] J-L. Loday, Spaces with finitely many nontrivial homotopy groups, $J$. Pure Appl. Algebra 24 (1982), no. 2, 179-202.

[McPa] S. MacLane, R. Paré, Coherence for bicategories and indexed categories, J. Pure Appl. Algebra 37 (1985), no. 1, 59-80.

[McWh] S. MacLane, J. H. C. Whitehead, On the 3-type of a complex, Proc. Nat. Acad. Sci. U.S.A. 36 (1950), 41-48.

[Ma] J. P. May, Simplicial objects in algebraic topology, Chicago Lectures in Mathematics, University of Chicago Press, Chicago, 1992.

[MoSe] I. Moerdijk and J. Svensson, Algebraic classification of equivariant homotopy 2-types, J. Pure Appl. Algebra 89 (1993), 187-216.

[No] B. Noohi, On weak maps between 2-groups, arxiv:math.CT/0506313, preprint, 2005.

[To] A. Tonks, On the Eilenberg-Zilber theorem for crossed complexes, $J$. Pure Appl. Algebra 179 (2003), 199-220.

[Wh] J. H. C. Whitehead, Combinatorial homotopy I, Bull. Amer. Math. Soc. 55 (1949), 213-245.

Behrang Noohi behrang@alum.mit.edu

Mathematics Department

Florida State University

208 Love Building

Tallahassee, FL 32306-4510

U.S.A.

This article is available at http://intlpress.com/HHA/v9/n1/a3 